\title{
BREGMAN DISTANCES, TOTALLY CONVEX FUNCTIONS, AND A METHOD FOR SOLVING OPERATOR EQUATIONS IN BANACH SPACES
}

\author{
DAN BUTNARIU AND ELENA RESMERITA
}

Received 25 July 2004; Accepted 6 April 2005

The aim of this paper is twofold. First, several basic mathematical concepts involved in the construction and study of Bregman type iterative algorithms are presented from a unified analytic perspective. Also, some gaps in the current knowledge about those concepts are filled in. Second, we employ existing results on total convexity, sequential consistency, uniform convexity and relative projections in order to define and study the convergence of a new Bregman type iterative method of solving operator equations.

Copyright (c) 2006 D. Butnariu and E. Resmerita. This is an open access article distributed under the Creative Commons Attribution License, which permits unrestricted use, distribution, and reproduction in any medium, provided the original work is properly cited.

\section{Introduction}

Let $X$ be a Banach space and $f: X \rightarrow(-\infty,+\infty]$ be a proper convex function. For any $x \in \operatorname{dom} f$ and $z \in X$ we denote by $f^{\circ}(x, z)$ the right-hand side derivative of $f$ at $x$ in the direction $z$, that is,

$$
f^{\circ}(x, z)=\lim _{t \searrow 0} \frac{f(x+t z)-f(x)}{t} .
$$

The function $D_{f}: \operatorname{dom} f \times \operatorname{dom} f \rightarrow[0,+\infty]$ given by

$$
D_{f}(y, x):=f(y)-f(x)-f^{\circ}(x, y-x)
$$

is called the Bregman distance with respect to $f$. Lev Bregman [20] has discovered an elegant and effective technique for the use of the function $D_{f}$ in the process of designing and analyzing feasibility and optimization algorithms. This opened a growing area of research in which Bregman's technique is applied in various ways in order to design and analyze iterative algorithms for solving not only feasibility and optimization problems, but also algorithms for solving variational inequalities, for approximating equilibria, for computing fixed points of nonlinear mappings and more (see $[3,6,12,16,20,23,24,26-$ $29,33,36,40,48,49,51,60,65]$, and the references therein). Although called a "distance" 
by Censor and Lent [35], the function $D_{f}$ is not a distance in the usual sense of the term: it is not always symmetric and may not satisfy the triangular inequality.

The main aim of this paper is to give an analytic perspective over the basic tools commonly used in the implementations of Bregman's technique and to show how these tools can be employed for building an algorithm of solving operator equations. An intrinsic feature of Bregman's technique of designing iterative algorithms consists of ensuring that the sequences $\left\{x^{k}\right\}_{k \in \mathbb{N}}$ these algorithms generate are contained in the domain of a convex function $f$ and have the property that $\lim _{k \rightarrow \infty} D_{f}\left(x^{k}, x\right)=0$ for points $x \in \operatorname{dom} f$ which are necessarily solutions of the problem the algorithm is supposed to solve. If the function $f$ is chosen such that, for any sequence $\left\{x^{k}\right\}_{k \in \mathbb{N}} \subset \operatorname{dom} f$ and any vector $x \in \operatorname{dom} f$, one has

$$
\lim _{k \rightarrow \infty} D_{f}\left(x^{k}, x\right)=0 \Longrightarrow \lim _{k \rightarrow \infty}\left\|x^{k}-x\right\|=0,
$$

then the convergence of the algorithm to a solution of the problem is guaranteed. That brings up the question of whether, and under which conditions, a convex function $f$ satisfies (1.3). Butnariu et al. [25] were the first to point out that totally convex functions, introduced in their paper under a different name, have that property. The function $f$ is called totally convex at a point $x \in \operatorname{dom} f$ if its modulus of total convexity at $x$, that is, the function $v_{f}(x, \cdot):[0,+\infty) \rightarrow[0,+\infty]$ defined by

$$
v_{f}(x, t)=\inf \left\{D_{f}(y, x): y \in \operatorname{dom} f,\|y-x\|=t\right\}
$$

is positive on $(0, \infty)$. Resmerita [62] made clear that the totally convex functions are the only convex functions satisfying (1.3). The importance of the notion of total convexity in applications stems primarily from its equivalence to condition (1.3). In the process of using totally convex functions in the convergence analysis of various algorithms, additional useful properties of those functions were discovered and some questions concerning the connections of totally convex functions with other classes of functions and with the geometry of the underlying space were raised. The results in these respects are scattered in literature, are placed in the specific restrictive contexts of the algorithmic procedures they were supposed to serve and are presented in various terminologies. One of the aims of this paper is to present, in a unified approach and in full generality, the properties of totally convex functions which proved to be of interest in the theory of various algorithms and, at the same time, to fill in several gaps in the existing knowledge about those functions (see Section 2).

In finite dimensional Banach spaces there is not much difference between totally convex and strictly convex functions. Any strictly convex function with closed domain which is continuous on $\operatorname{dom} f$ is totally convex. However, in infinite dimensional spaces, totally convex functions constitute a very special class of strictly convex functions and identifying totally convex functions with specific features required in applications is quite complicated. In an infinite dimensional setting, for reasons related to effective computability, the functions $\|\cdot\|^{p}$ with $p>1$ are among the most likely candidates to be used in the build up of Bregman type algorithms. Therefore, it is interesting to know which are the Banach spaces in which the functions $\|\cdot\| p$ with $p>1$ are totally convex. Those spaces 
were termed locally totally convex spaces in [62]. The locally uniformly convex spaces are among the locally totally convex spaces. In Section 3 we give geometric characterizations of locally totally convex spaces. These characterizations lead us to the conjecture that there exist Banach spaces which are locally totally convex without being locally uniformly convex. However, we do not have any example to support this claim.

A notion strongly related to that of total convexity is that of projection onto a closed convex set relative to a totally convex function (see Section 4). This notion is a straightforward generalization of the concept of "generalized projection" due to Alber $[2,3]$. The projection relative to $f$ onto a closed convex set $K$ is a monotone operator from $X$ to its dual $X^{*}$. In some circumstances, projection operators relative to the totally convex function $f$ can be seen as extensions of Bregman projection operators to larger sets. Bregman projections are the corner stones of a plethora of algorithms (see $[28,36]$ and the references therein). Relative projections were successfully used for designing and analyzing various algorithms by Alber et al. [4, 6-9]. The algorithm for solving equations in Banach spaces presented in Section 5, illustrates the way in which projections relative to totally convex functions can be used for that purpose. It is a procedure for finding solutions of the problem

$$
A x=0, \quad x \in \Omega,
$$

where $A: X \rightarrow X^{*}$ is a given operator and $\Omega$ is a closed convex subset of $X$. This algorithm is a refinement of a procedure originating in Landweber's [52] method of finding minimum norm solutions of linear equations and in Polyak's [58] method of finding zeros for subgradients of convex functions. That procedure was subsequently extended by Bruck [21, 22], Reich [59] and by Alber [4] to a method of solving operatorial equations and variational inequalities in Banach spaces. The general iterative formula on which the algorithm is based is

$$
y^{k+1}=P_{\Omega}^{f}\left[f^{\prime}\left(y^{k}\right)-\alpha A y^{k}\right]
$$

where $\alpha \in(0, \infty), f^{\prime}$ stands for the Gâteaux derivative of $f$ and $P_{\Omega}^{f}$ stands for the projection operator onto $\Omega$ relative to $f$. We are proving convergence of this algorithm when applied to classes of operators $A$ satisfying certain monotonicity type properties we call inverse-monotonicity and strong-inverse- monotonicity relative to a totally convex function $f$. These properties require that the operator $A_{\alpha}^{f} x=f^{*^{\prime}}\left[f^{\prime}(x)-\alpha A x\right]$, where $f^{*}$ is the Fenchel conjugate of $f$, should satisfy some nonexpansivity type properties conditioned by the function $f$. In Hilbert spaces, such requirements are commonly satisfied by the operators $A$ whose (set valued) inverses are strongly monotone because they are inverse (strongly) monotone relative to $f=(1 / 2)\|\cdot\|^{2}$. In this particular case $P_{\Omega}^{f}$ is exactly the metric projection operator $P_{\Omega}$ and the algorithmic procedure is $y^{k+1}=$ $P_{\Omega}\left(y^{k}-\alpha A y^{k}\right)$. In non-Hilbertian spaces, inverse-strong-monotonicity of $A$ is less useful in order to ensure convergence of the algorithm $y^{k+1}=P_{\Omega}\left(y^{k}-\alpha A y^{k}\right)$ mostly due to the lack of nonexpansivity of $P_{\Omega}$. In this situation we take advantage of the nonexpansivity like properties of $P_{\Omega}^{f}$ when $f$ is totally convex, and of the presumed inverse-monotonicity of $A$ relative to $f$, in order to ensure convergence of our algorithm. 
The new results proven in this paper are as follows. Theorems 2.10 and 2.14 elucidate the connections between total convexity and uniform convexity in infinite and, respectively, in finite dimensional spaces. Theorem 3.3 is a geometric characterization of the Banach spaces in which the function $\|\cdot\|^{2}$ is totally convex at any point. If $X$ is reflexive, then this result can be interpreted as another characterization of the $E$-spaces introduced by Fan and Glicksberg [44]. Theorem 4.5 establishes continuity of the projections relative to a totally convex functions with respect to the Mosco convergence. Theorem 4.7 shows a way of computing relative projections on hyperplanes and half-spaces. Theorems 5.4 and 5.7 are convergence results for the algorithm described above.

\section{Totally convex functions}

2.1. We start our presentation of the totally convex functions by summarizing some basic properties of the modulus of total convexity. They were stated in $[28,48]$ under the additional assumption that $x$ is a point in the algebraic interior of $\operatorname{dom} f$. A careful analysis of the proofs given there shows that this assumption is superfluous.

Proposition 2.1. Let $X$ be a Banach space and $f: X \rightarrow(-\infty,+\infty]$ be a convex function such that $\operatorname{int}(\operatorname{dom} f) \neq \varnothing$. If $x \in \operatorname{dom} f$, then

(i) the domain of $\nu_{f}(x, \cdot)$ is an interval $\left[0, \tau_{f}(x)\right)$ or $\left[0, \tau_{f}(x)\right]$ with $\tau_{f}(x) \in(0,+\infty]$, if $\partial f(x) \neq \varnothing$, and it is $\{0\}$, otherwise;

(ii) if $c \geq 1$ and $t \geq 0$, then $v_{f}(x, c t) \geq c v_{f}(x, t)$;

(iii) the function $v_{f}(x, \cdot)$ is superadditive, that is, for any $s, t \in[0,+\infty)$, one has $v_{f}(x, s+$ $t) \geq v_{f}(x, s)+v_{f}(x, t)$

(iv) the function $v_{f}(x, \cdot)$ is nondecreasing; it is strictly increasing on its domain if and only if $f$ is totally convex at $x$;

(v) the following equality holds

$$
v_{f}(x, t)=\inf \left\{D_{f}(y, x): y \in \operatorname{dom} f,\|y-x\| \geq t\right\} .
$$

Proof. As mentioned at the beginning of this section, the statements (i)-(iv) are more general than the ones in [28, page 18], but the proofs are the same. We now show that (v) also holds. To this end, denote by $v_{f}^{\#}(x, t)$ the right-hand side of (2.1). Clearly,

$$
v_{f}^{\#}(x, t) \leq v_{f}(x, t)
$$

for all $t \geq 0$ and the equality holds for $t=0$. Suppose that $t>0$. We show that for any $y \in \operatorname{dom} f$ having $\|y-x\| \geq t$ there exists $y^{\prime} \in \operatorname{dom} f$ such that $\left\|y^{\prime}-x\right\|=t$ and

$$
D_{f}(y, x) \geq D_{f}\left(y^{\prime}, x\right) .
$$

Since $f$ is convex, the function $D_{f}(\cdot, x)$ is also convex. Then,

$$
D_{f}(\alpha y+(1-\alpha) x, x) \leq \alpha D_{f}(y, x)+(1-\alpha) D_{f}(x, x)=\alpha D_{f}(y, x) .
$$

Now, take $\alpha=t /\|y-x\|$ in (2.4). This gives exactly (2.3) for $y^{\prime}=y_{\alpha}$ and, clearly, one has $\left\|y_{\alpha}-x\right\|=t$. The proof is complete. 
2.2. Total convexity is a strong form of strict convexity, as shown by the following result. Proposition 2.2 (cf. [28, page 34]). (i) If $f$ is totally convex at any $x \in \operatorname{dom} f$, then it is strictly convex on this set.

(ii) If $\operatorname{dim} X<+\infty, \operatorname{dom} f$ is closed and the function $f$ restricted to $\operatorname{dom} f$ is continuous and strictly convex, then $f$ is totally convex at any $x \in \operatorname{dom} f$.

2.3. The following features of the moduli of total convexity give rules for generating totally convex functions by aggregating other totally convex functions.

Proposition 2.3 (cf. [28, page 28]). (i) Let $f_{1}, \ldots, f_{m}: X \rightarrow(-\infty,+\infty]$ be totally convex functions with domains $D_{1}, \ldots, D_{m}$, respectively, such that $D=\cap_{i=1}^{m} D_{i} \neq \varnothing$. Then, for any $m$ nonnegative real numbers $c_{1}, \ldots, c_{m}$ with $\sum_{i=1}^{m} c_{i}>0$, the function $h=\sum_{i=1}^{m} c_{i} f_{i}$ is totally convex and, for any $x \in D$ and for all $t \in[0,+\infty)$,

$$
\nu_{h}(x, t) \geq \sum_{i=1}^{m} c_{i} \nu_{f_{i}}(x, t) ;
$$

(ii) If $f$ is totally convex and lower semicontinuous with open domain D and $\phi$ is a real convex function, differentiable and strictly increasing on an open interval which contains $f(D)$, then the function $g: X \rightarrow(-\infty,+\infty]$ defined by $g=\phi \circ f($ with $g(x)=+\infty$ for $x \notin D)$ is totally convex and

$$
v_{g}(x, t) \geq \phi^{\prime}(f(x)) \cdot v_{f}(x, t)
$$

for any $x \in D$ and for all $t \geq 0$.

2.4. Here are other total convexity criteria which allow building totally convex function on product spaces.

Proposition 2.4 (cf. [48]). Let $X_{1}, X_{2}$ be real Banach spaces, $f_{i}: X_{i} \rightarrow(-\infty,+\infty], i=1,2$, two proper convex lower semicontinuous functions whose domains have nonempty interiors. Define the function $f: X_{1} \times X_{2} \rightarrow(-\infty,+\infty]$ by $f\left(x_{1}, x_{2}\right)=f_{1}\left(x_{1}\right)+f_{2}\left(x_{2}\right)$. Then

(i) For any $x=\left(x_{1}, x_{2}\right) \in \operatorname{dom} f$, such that $\partial f(x) \neq \varnothing$, the domain of $\nu_{f}(x, \cdot)$ is an interval $\left[0, \tau_{f}(x)\right)$ or $\left[0, \tau_{f}(x)\right]$ with $0 \leq \tau_{f}(x)=\tau_{f_{1}}\left(x_{1}\right)+\tau_{f_{2}}\left(x_{2}\right) \leq \infty$, where

$$
\tau_{f_{i}}\left(x_{i}\right)=\sup \left\{t: t \in \operatorname{dom} v_{f_{i}}\left(x_{i}, \cdot\right)\right\}, \quad i=1,2 .
$$

Moreover,

$$
v_{f}(x, t)=\inf \left\{v_{f_{1}}\left(x_{1}, s\right)+v_{f_{2}}\left(x_{2}, t-s\right): s \in[0, t]\right\} .
$$

If $\partial f(x)=\varnothing$, then $\operatorname{dom} v_{f}(x, \cdot)=\{0\}$.

(ii) For any $x=\left(x_{1}, x_{2}\right) \in \operatorname{dom} f$ and $t \in \operatorname{dom} v_{f}(x, \cdot)$,

$$
v_{f}(x, t) \geq \min \left\{v_{f_{i}}\left(x_{i}, \frac{t}{2}\right)\right\}, \quad i=1,2 .
$$

Therefore, if both functions $f_{i}$ are totally convex, then $f$ is totally convex. 
2.5. The following characterization of total convexity makes clear why functions with this property are useful in convergence analysis of many algorithms: $D_{f}$-convergence of a sequence $\left\{y^{n}\right\}_{n \in \mathbb{N}}$ to $x$, that is, the fact that $\left\{D_{f}\left(y^{n}, x\right)\right\}_{n \in \mathbb{N}}$ converges to zero (see [15]) implies convergence in norm of $\left\{y^{n}\right\}_{n \in \mathbb{N}}$ to $x$.

Proposition 2.5 (cf. [62]). Let $X$ be a Banach space and $f: X \rightarrow(-\infty,+\infty]$ be a convex function. If $x \in \operatorname{dom} f$, then the following statements are equivalent:

(i) the function $f$ is totally convex at $x$;

(ii) for any sequence $\left\{y^{n}\right\}_{n \in \mathbb{N}} \subseteq \operatorname{dom} f$,

$$
\lim _{n \rightarrow \infty} D_{f}\left(y^{n}, x\right)=0 \Longrightarrow \lim _{n \rightarrow \infty}\left\|y^{n}-x\right\|=0 ;
$$

(iii) For any sequence $\left\{y^{n}\right\}_{n \in \mathbb{N}} \subseteq \operatorname{dom} f$,

$$
\liminf _{n \rightarrow \infty} D_{f}\left(y^{n}, x\right)=0 \Longrightarrow \liminf _{n \rightarrow \infty}\left\|y^{n}-x\right\|=0 ;
$$

(iv) for any sequence $\left\{y^{n}\right\}_{n \in \mathbb{N}} \subseteq \operatorname{dom} f$,

$$
\lim _{n \rightarrow \infty} D_{f}\left(y^{n}, x\right)=0 \Longrightarrow \liminf _{n \rightarrow \infty}\left\|y^{n}-x\right\|=0 ;
$$

(v) for any $\varepsilon>0$, there exists $\delta>0$ such that if $y \in \operatorname{dom} f$ and $D_{f}(y, x) \leq \delta$, then $\| x-$ $y \| \leq \varepsilon$.

2.6. Characteristic properties of totally convex functions were given in $[31,32,62]$. Denote by $\mathscr{F}$ the set of functions $\psi:[0,+\infty) \rightarrow[0,+\infty]$ which satisfy the conditions below:

(i) int $\operatorname{dom} \psi \neq \varnothing$;

(ii) $\psi$ is convex and lower semicontinuous;

(iii) $\psi(0)=0$ and $\psi(t)>0$ whenever $t>0$.

The following characterization appears in [31].

Proposition 2.6. Suppose that $x \in \operatorname{dom} f$ and $\partial f(x) \neq \varnothing$. The function $f$ is totally convex at $x$ if and only if there exists a function $\varphi \in \mathscr{F}$ such that, for any $y \in X$, one has

$$
f(y)-f(x) \geq f^{\circ}(x, y-x)+\varphi(\|y-x\|) .
$$

2.7. We list below some special features which make totally convex functions of interest in many applications. To this end, recall (see [69, Section 3.3]) that the pseudo-conjugate of a function $\varphi \in \mathscr{F}$, is the lower semicontinuous convex function $\varphi^{\#}:[0, \infty) \rightarrow[0, \infty]$ defined by

$$
\varphi^{\#}(t)=\sup \{s t-\varphi(s): s \geq 0\}
$$

Proposition 2.7 (cf. [31]). If the function $f$ is totally convex at $x \in \operatorname{dom} f$ and if $x^{*} \in$ $\partial f(x)$, then $x^{*} \in \operatorname{int}\left(\operatorname{dom} f^{*}\right)$ and any of the following equivalent conditions are satisfied:

(i) there exists $\varphi \in \mathscr{F}$ such that, for any $y \in X$, one has

$$
f(y)-f(x) \geq\left\langle x^{*}, y-x\right\rangle+\varphi(\|y-x\|)
$$




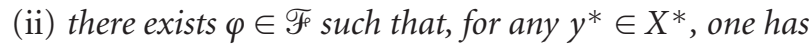

$$
f^{*}\left(y^{*}\right)-f^{*}\left(x^{*}\right) \leq\left\langle y^{*}-x^{*}, x\right\rangle+\varphi^{\#}\left(\left\|y^{*}-x^{*}\right\|\right) ;
$$

(iii) the function $f^{*}$ is Fréchet differentiable at $x^{*}$.

If, in addition, $f$ is lower semicontinuous, then these conditions are also equivalent to each of the following requirements:

(iv) there exists $\varphi \in \mathscr{F}$ such that, for any pair $\left(y, y^{*}\right) \in X \times X^{*}$ with $y^{*} \in \partial f(y)$, one has

$$
\left\langle y^{*}-x^{*}, y-x\right\rangle \geq \varphi(\|y-x\|)
$$

(v) there exists a nondecreasing function $\theta:[0,+\infty) \rightarrow[0,+\infty]$ with $\lim _{t \backslash 0} \theta(t)=0$ such that, for any pair $\left(y, y^{*}\right) \in X \times X^{*}$ with $y^{*} \in \partial f(y)$, one has

$$
\|y-x\| \leq \theta\left(\left\|y^{*}-x^{*}\right\|\right)
$$

2.8. Under additional assumptions on the function $f$, one obtains the following characterization of the total convexity of $f$ at a point.

Proposition 2.8 (cf. [31]). Suppose that $f$ is continuous at the point $x \in \operatorname{int}(\operatorname{dom} f)$. Then, the following conditions are equivalent:

(i) the function $f$ is totally convex at $x$;

(ii) there exists $\varphi \in \mathscr{F}$ such that, for any $y \in X$ and for any $x^{*} \in \partial f(x)$ one has

$$
f(y)-f(x) \geq\left\langle x^{*}, y-x\right\rangle+\varphi(\|y-x\|)
$$

(iii) there exists $\varphi \in \mathscr{F}$ such that, for any $y^{*} \in X^{*}$ and for any $x^{*} \in \partial f(x)$ one has

$$
f^{*}\left(y^{*}\right)-f^{*}\left(x^{*}\right) \leq\left\langle y^{*}-x^{*}, x\right\rangle+\varphi^{\#}\left(\left\|y^{*}-x^{*}\right\|\right) ;
$$

(iv) $\partial f(x) \subseteq \operatorname{int}\left(\operatorname{dom}\left(f^{*}\right)\right)$ and the function $f^{*}$ is uniformly Fréchet differentiable on $\partial f(x)$.

If, in addition, the function $f$ is lower semicontinuous, then these conditions are equivalent to each of the following requirements:

(v) There exists $\varphi \in F$ such that, for any $x^{*} \in \partial f(x)$ and for any pair $\left(y, y^{*}\right) \in X \times X^{*}$ with $y^{*} \in \partial f(y)$, one has

$$
\left\langle y^{*}-x^{*}, y-x\right\rangle \geq \varphi(\|y-x\|)
$$

(vi) There exists a nondecreasing function $\theta:[0,+\infty) \rightarrow[0,+\infty]$ with $\lim _{t \backslash 0} \theta(t)=0$ such that, for any pair $\left(y, y^{*}\right) \in X \times X^{*}$ with $y^{*} \in \partial f(y)$, one has

$$
\|y-x\| \leq \theta\left(\left\|y^{*}-x^{*}\right\|\right)
$$

2.9. According to Proposition 2.2, in spaces of finite dimension any strictly convex function with closed domain, which is continuous on its domain, is totally convex at any point 
of its domain. In infinite dimensional Banach spaces the distinction between strict convexity and total convexity is more drastic as we explain below. We start by reviewing the relationships between the modulus of total convexity and several moduli of uniform convexity. Recall (see, e.g., [31, 67], and [69, page 203]) that the modulus of uniform convexity of $f$ at $x \in \operatorname{dom} f$ is the function $\mu_{f}(x, \cdot):[0,+\infty) \rightarrow[0,+\infty]$ defined by

$$
\mu_{f}(x, t)=\inf \left\{f(x)+f(y)-2 f\left(\frac{x+y}{2}\right): y \in \operatorname{dom} f,\|y-x\|=t\right\} .
$$

The function $f$ is called uniformly convex at $x$ if $\mu_{f}(x, t)>0$, whenever $t>0$. by

The modulus of uniform convexity of $f$ is the function $\mu_{f}:[0,+\infty) \rightarrow[0,+\infty]$ defined

$$
\mu_{f}(t)=\inf \left\{f(x)+f(y)-2 f\left(\frac{x+y}{2}\right): x, y \in \operatorname{dom} f,\|y-x\|=t\right\} .
$$

The function $f$ is called uniformly convex if $\mu_{f}(t)>0$, whenever $t>0$.

It was shown in [28] that uniform convexity of a function at a point implies total convexity at that point, while the converse is not true. The last fact is illustrated by the function $g: \ell^{1} \rightarrow \mathbb{R}$ given by

$$
g(x)=\sum_{k=1}^{\infty}\left|x_{k}\right|^{1+1 / k} .
$$

This function is totally convex at any point of the convex set

$$
C=\left\{x \in \ell^{1}: \limsup _{k \rightarrow \infty}\left|x_{k}\right|^{1 / k}<1\right\}
$$

but it is not uniformly convex at any point of $\ell^{1}$. However, under additional hypothesis, one has the following result.

Proposition 2.9 (cf. [31]). Suppose that $f$ is lower semicontinuous. If $f$ is Fréchet differentiable at $x \in \operatorname{dom} f$, then $f$ is totally convex at $x$ if and only if $f$ is uniformly convex at $x$.

2.10. In order to have a better understanding of the connections between the notion of total convexity and that of uniform convexity, recall that the modulus of uniformly strict convexity of $f$ on the set $E \subseteq X$ is the function $\mu_{f}(E, \cdot):[0,+\infty) \rightarrow[0,+\infty]$ given by

$$
\mu_{f}(E, t)=\inf \left\{\mu_{f}(x, t): x \in E \cap \operatorname{dom} f\right\} .
$$

The function $f$ is said to be uniformly strictly convex on bounded sets (cf. [10]) if for each nonempty bounded subset $E$ of $X, \mu_{f}(E, t)>0$ for all $t \in(0,+\infty)$. Also, recall (cf. [28]) that the modulus of total convexity of the function $f$ on the set $E$ is the function $\nu_{f}(E, \cdot):[0,+\infty) \rightarrow[0,+\infty]$ given by

$$
v_{f}(E, t)=\inf \left\{\nu_{f}(x, t): x \in E \cap \operatorname{dom} f\right\} .
$$


The function $f$ is called totally convex on bounded sets if $\nu_{f}(E, t)$ is positive for any nonempty bounded set $E$ and any $t>0$. It immediately results from Proposition 2.1 that, if $f$ is totally convex on bounded sets and the set $E$ is nonempty and bounded, then $v_{f}(E, c t) \geq c v_{f}(E, t)$ for any $c \geq 1$ and $t \geq 0$. Consequently, in this case, the function $v_{f}(E, \cdot)$ is strictly increasing on $[0,+\infty)$. The relevance of the concept of total convexity on bounded sets in convergence analysis of various algorithms (see $[15,28,31,33,36]$ ) stems from the fact that functions which are totally convex on bounded sets are sequentially consistent. Recall that the function $f$ is called sequentially consistent if for any two sequences $\left\{x^{k}\right\}_{k \in \mathbb{N}}$ and $\left\{y^{k}\right\}_{k \in \mathbb{N}}$ in $X$ such that the first is bounded,

$$
\lim _{k \rightarrow \infty} D_{f}\left(y^{k}, x^{k}\right)=0 \Longrightarrow \lim _{k \rightarrow \infty}\left\|x^{k}-y^{k}\right\|=0 .
$$

We follow [69] and call the function $f$ uniformly convex on bounded sets if for each positive real numbers $r$ and $t$ the function $f_{r}=f+\iota_{B(0, r)}$ has $\mu_{f_{r}}(X, t)>0$, where $\iota_{B(0, r)}$ stands for the indicator function of the closed ball of center 0 and radius $r$ in $X$.

The following result shows the connections between sequential consistency, uniform convexity on bounded sets, total convexity on bounded sets and uniformly strict convexity on bounded sets. One should note that, for lower semicontinuous functions, the sequential consistency, the total convexity on bounded sets and the uniform convexity on bounded sets are equivalent. Uniform strict convexity seems to be a stronger condition than sequential consistency, although we do not have examples of functions which are sequentially consistent without being uniformly strictly convex.

Theorem 2.10. Let $f: X \rightarrow(-\infty,+\infty]$ be a convex function whose domain contains at least two points. The following statements hold true:

(i) the function $f$ is sequentially consistent if and only if it is totally convex on bounded sets;

(ii) if $f$ is also lower semicontinuous, then $f$ is sequentially consistent if and only if $f$ is uniformly convex on bounded sets;

(iii) if $f$ is uniformly strictly convex on bounded sets, then it is sequentially consistent and the converse implication holds when $f$ is lower semicontinuous, Frechet differentiable on its domain and the Fréchet derivative $f^{\prime}$ is uniformly continuous on bounded sets.

Proof. The statements (i) and (ii) were proved in [31, Proposition 4.2]. In analyzing the proof of that result one should take into account that the definition of uniform convexity on bounded sets given in [31, Section 4.1] is incorrectly formulated. It is congruent with what we call here uniformly strict convexity on bounded sets. However, the notion of uniform convexity on bounded sets de facto used in the paper [31] is that occurring here. The fact that uniformly strict convexity on bounded sets implies total convexity on bounded sets follows immediately from [28, Proposition 1.2.5]. We show next that if $f$ is lower semicontinuous, totally convex on bounded sets, Fréchet differentiable on $\operatorname{dom} f$ and its Fréchet derivative $f^{\prime}$ is uniformly continuous on bounded sets, then $f$ is also uniformly strictly convex on bounded sets. Note that $\operatorname{dom} f$ is open because $f$ is Fréchet differentiable on it. Let $E$ be a bounded subset of $X$ such that $E \cap \operatorname{dom} f \neq \varnothing$. Suppose that $t>0$ is a number such that $v_{f}(E, t / 2)$ is finite. Then, by the total convexity of $f$ on bounded sets, we deduce that $v_{f}(E, t / 2)>0$. Since $f^{\prime}$ is uniformly continuous on the 
10 Bregman distances, total convexity, operator equations

bounded set $E \cap \operatorname{dom} f$, there exists a number $\tau>0$ such that, for any $x, z \in E \cap \operatorname{dom} f$, one has

$$
\|z-x\| \leq \tau \Longrightarrow\left\|f^{\prime}(z)-f^{\prime}(x)\right\| \leq \frac{1}{t} \nu_{f}\left(E, \frac{t}{2}\right)
$$

Fix $x \in E \cap \operatorname{dom} f$ arbitrarily. For any $y \in X$ with $\|y-x\|=t / 2$ one has

$$
f(y) \geq f(x)+\left\langle f^{\prime}(x), y-x\right\rangle+v_{f}\left(E, \frac{t}{2}\right)
$$

Let $\alpha \in(0,1)$ be such that $1-\alpha<2 \tau / t$ and assume that $u \in \operatorname{dom} f$ is such that $\|u-x\|=$ $t$. Define

$$
w=x+\frac{1-\alpha}{2}(u-x)
$$

Clearly, $w \in \operatorname{dom} f$ because it is a convex combination of $x, u \in \operatorname{dom} f$. Note that

$$
\|w-x\|=\frac{1-\alpha}{2}\|u-x\|=\frac{1-\alpha}{2} t \leq \tau
$$

and this implies that

$$
\left\|f^{\prime}(w)-f^{\prime}(x)\right\| \leq \frac{1}{t} v_{f}\left(E, \frac{t}{2}\right)
$$

because of (2.30). Obviously, one has

$$
\begin{gathered}
f(x) \geq f(w)+\left\langle f^{\prime}(w), x-w\right\rangle=f(w)+\frac{1-\alpha}{2}\left\langle f^{\prime}(w), x-u\right\rangle, \\
\frac{1}{2}(x+u)=\frac{\alpha}{\alpha+1} u+\frac{1}{\alpha+1} w .
\end{gathered}
$$

From (2.36) one deduces that

$$
\frac{\alpha}{\alpha+1} f(u)+\frac{1}{\alpha+1} f(w) \geq f\left(\frac{1}{2}(x+u)\right)
$$

that is,

$$
\alpha f(u)+f(w) \geq(1+\alpha) f\left(\frac{1}{2}(x+u)\right)
$$

Summing up this inequality and (2.35) one gets

$$
f(x)+\alpha f(u) \geq(1+\alpha) f\left(\frac{1}{2}(x+u)\right)+\left\langle f^{\prime}(w), \frac{1-\alpha}{2}(x-u)\right\rangle .
$$


Consequently,

$$
\begin{aligned}
f(x) & +f(u)-2 f\left(\frac{1}{2}(x+u)\right) \\
& \geq \frac{1-\alpha}{\alpha}\left[f\left(\frac{1}{2}(x+u)\right)-f(x)+\frac{1}{2}\left\langle f^{\prime}(w), x-u\right\rangle\right] \\
& \geq \frac{1-\alpha}{\alpha}\left[v_{f}\left(E, \frac{t}{2}\right)+\frac{1}{2}\left\langle f^{\prime}(x), u-x\right\rangle+\frac{1}{2}\left\langle f^{\prime}(w), x-u\right\rangle\right] \\
& \geq \frac{1-\alpha}{\alpha}\left[v_{f}\left(E, \frac{t}{2}\right)-\frac{1}{2}\left\|f^{\prime}(x)-f^{\prime}(w)\right\|\|u-x\|\right] \\
& =\frac{1-\alpha}{\alpha}\left[v_{f}\left(E, \frac{t}{2}\right)-\frac{t}{2}\left\|f^{\prime}(x)-f^{\prime}(w)\right\|\right] \\
& \geq \frac{1-\alpha}{\alpha}\left[v_{f}\left(E, \frac{t}{2}\right)-\frac{t}{2} \frac{v_{f}(E, t / 2)}{t}\right],
\end{aligned}
$$

where the last inequality results from (2.34). Hence, one has

$$
f(x)+f(u)-2 f\left(\frac{1}{2}(x+u)\right) \geq \frac{1-\alpha}{2 \alpha} v_{f}\left(E, \frac{t}{2}\right),
$$

for all $u \in X$ with $\|u-x\|=t$ and this implies that

$$
\mu_{f}(x, t) \geq \frac{1-\alpha}{2 \alpha} v_{f}\left(E, \frac{t}{2}\right)>0 .
$$

Since this inequality holds for any $x \in E \cap \operatorname{dom} f$, the proof is complete.

2.11. The following property of totally convex functions on bounded sets was used in the study of generic fixed point properties of some classes of operators done in [32]. Although stated in [32, Lemma 2.1] for uniformly convex functions, the proof is done in fact for functions which are totally convex on bounded sets.

Proposition 2.11. If the function $f$ is totally convex on bounded sets, then for each $x \in$ $\operatorname{dom} f$ and each $\epsilon>0$, there exists $\delta>0$ such that if $y, z \in \operatorname{dom} f,\|y-x\| \leq \delta$ and $D_{f}(z$, $y) \leq \delta$, then $\|x-z\| \leq \epsilon$.

2.12. In finite dimensional spaces, one of the most useful totally convex functions is the negentropy, that is, the negative of the Boltzmann-Shannon entropy (see [36]). Its extension to $\mathscr{L}^{1}(\Omega)$, when $\Omega$ is a nonempty bounded and measurable subset of $\mathbb{R}^{d}$, is the function $f: \mathscr{L}^{1}(\Omega) \rightarrow(-\infty,+\infty]$ defined by

$$
f(x)= \begin{cases}\int_{\Omega} x(t) \ln x(t) d t & \text { if } x \geq 0 \text { a.e., } x \ln x \in \mathscr{L}^{1}(\Omega), \\ +\infty & \text { otherwise. }\end{cases}
$$

This function is used in methods which aim at reconstructing a function from a finite number of moments (see $[18,19]$ ) as well as in regularization methods for ill-posed inverse problems (see, e.g., $[41,43]$ ). Note that the domain of the negentropy $f$ is not equal 
to $\mathscr{L}_{+}^{1}(\Omega)$, but it is a much smaller set. For instance, if $\Omega=[0,1]$, then the nonnegative function $x$ defined by $x(t)=1 / t(\ln t-1)^{2}$ for $t \in(0,1]$ and $x(0)=0$, belongs to $\mathscr{L}^{1}(\Omega)$, while $x \ln x$ does not, that is, $x$ is not contained in $\operatorname{dom} f$. This implies that, contrary to what is claimed in [13, Remark 5.7], the domain of the function defined by

$$
I_{\psi}(x):=f(x)-\|x\|_{\mathscr{L}^{1}(\Omega)}
$$

for any $x \in \mathscr{L}_{+}^{1}(\Omega)$, which obviously equals that of the negentropy, is not $\mathscr{L}_{+}^{1}(\Omega)$, but a smaller set.

The interior of the domain of $f$ is empty since the interior of $\mathscr{L}_{+}^{1}(\Omega)$ is so. Thus, the function is not differentiable, while its directional derivative $f^{\circ}(x, y-x)$ exists and belongs to $[-\infty,+\infty)$, for all $x, y \in \operatorname{dom} f$. The following result shows that the extended negentropy preserves the most basic properties which make its finite counterpart of much interest in the convergence analysis of algorithms in $\mathbb{R}^{n}$.

Proposition 2.12. The function $f$ defined by (2.43) has the following properties:

(i) for any $x, y \in \operatorname{dom} f$, one has

$$
\|y-x\|^{2} \leq\left(\frac{2}{3}\|y\|+\frac{4}{3}\|x\|\right) D_{f}(y, x)
$$

(ii) for any $x \in \operatorname{dom} f$ and $\alpha>0$, the following level sets are weakly compact in $\mathscr{L}^{1}(\Omega)$ :

$$
\left\{y \in \operatorname{dom} f: D_{f}(y, x) \leq \alpha\right\}
$$

(iii) the function $f$ is totally convex on bounded sets and, for any nonempty bounded set $E \subseteq \operatorname{dom} f$, there exist two positive real numbers $a$ and $b$ such that, for any number $t \geq 0$, we have

$$
v_{f}(E, t) \geq \frac{t^{2}}{a t+b}
$$

(iv) the function $f$ is uniformly convex on bounded sets.

Proof. (i) See [18]. (ii) See [42, Chapter 8, Theorem 1.3]. (iii) it follows immediately from (i). (iv) results from Theorem 2.10 .

2.13. Total convexity is a somewhat stronger form of essentially strict convexity. Recall, (cf. [13]), that the function $f$ is essentially strictly convex if $(\partial f)^{-1}$ is locally bounded on its domain and $f$ is strictly convex on every convex subset of $\operatorname{dom}(\partial f)$. This notion is of interest in the theory of some classes of fixed point algorithms $([12,13])$; it was introduced by Rockafellar [63] in $\mathbb{R}^{n}$ and was further generalized and studied by Bauschke et al. [13] in the context of infinite dimensional Banach spaces. The following result, establishing the connection between totally convex and essentially strictly convex functions, is a reformulation of a similar one from [62] where it was proved under the additional, 
but superfluous, hypotheses that $\operatorname{dom}(\partial f)$ is convex. Since total convexity of a function is a local notion, convexity of the set on which total convexity is discussed is not necessary.

Proposition 2.13. Suppose that $X$ is reflexive and that $f$ is a proper convex lower semicontinuous function which is totally convex at any point of $\operatorname{dom}(\partial f)$. Then $f$ is essentially strictly convex.

2.14. The converse implication in Proposition 2.13 is not true. To see that, let $f=$ $(1 / 2)\|\cdot\|^{2}$ and suppose that the space $X$ is strictly convex and reflexive. Then, according to [13, Definition 5.2], the function $f$ is essentially strictly convex. However, the function $f$ may not be totally convex as happens in the case when $X$ is the space contained in Vanderwerff's example (see [15, page 3]). That space is strictly convex and reflexive but does not have the Kadec-Klee property; in such a situation $f$ is not totally convex, as follows from Proposition 3.2 below. In finite dimensional Banach spaces, essential strict convexity seems to be slightly different from total convexity. In this respect, it is interesting to note that the most useful essentially strictly convex functions in $\mathbb{R}^{n}$ (see, for instance, [12]) are also totally convex. Moreover, any function which has closed domain and which is strictly convex and continuous on its domain, as well as any strictly convex function whose domain is the entire space is totally convex (cf. [28, Proposition 1.2.6]) and essentially strictly convex (cf. Proposition 2.13). On one hand, we do not have any example of a function which simultaneously satisfies the assumptions of Proposition 2.13 and is essentially strictly convex without being totally convex. On the other hand, we do not have a proof for the equivalence of the two notions even in finite dimensional Banach spaces except for the case $\operatorname{dom} f=\mathbb{R}^{n}$ discussed below.

2.15. It was noted in Section 2.9 that, in general, strict convexity and total convexity are not equivalent notions. However, if $\operatorname{dom} f=\mathbb{R}^{n}$, then $f$ is totally convex at any point of $\mathbb{R}^{n}$ if and only if it is strictly convex as follows from Proposition 2.2. Moreover, for functions $f$ having $\operatorname{dom} f=\mathbb{R}^{n}$, the strict convexity is a property equivalent to the uniform convexity on bounded sets. This may be already known, but we do not have any reference for it. In the case of functions $f$ defined and differentiable on the whole space $\mathbb{R}^{n}$, the equivalence between strict convexity and uniform convexity on bounded sets was implicitly shown in [45, Lemma 1.1, page 60]. Without requiring differentiability of $f$, but in the case of functions $f$ with $\operatorname{dom} f=\mathbb{R}$, the same equivalence is proved in [69, Proposition 3.6.5]. Our next statement shows that for functions $f$ which are finite everywhere on $\mathbb{R}^{n}$, uniform convexity on bounded sets is equivalent to strict convexity.

Theorem 2.14. Let $f: \mathbb{R}^{n} \rightarrow \mathbb{R}$ be a convex function. Then the following conditions are equivalent:

(i) $f$ is strictly convex;

(ii) $f$ is essentially strictly convex;

(iii) $f$ is totally convex at any $x \in \mathbb{R}^{n}$;

(iv) $f$ is uniformly convex at any $x \in \mathbb{R}^{n}$;

(v) $f$ is totally convex on bounded sets;

(vi) $f$ is sequentially consistent;

(vii) $f$ is uniformly convex on bounded sets. 
Proof. The equivalences $(\mathrm{v}) \Leftrightarrow(\mathrm{vi}) \Leftrightarrow$ (vii) result from Theorem 2.10 . The implications $(\mathrm{v}) \Rightarrow$ (iv) $\Rightarrow$ (iii) $\Rightarrow$ (ii) follow from Theorem 2.10, [28, Proposition 1.2.5] and Proposition 2.13, while the implication (ii) $\Rightarrow$ (i) follows from [13, Theorem 5.11]. We prove here that (i) $\Rightarrow$ (vii). To this end, one applies [69, Theorem 3.5.10]. According to this theorem, it is sufficient to show that if $E$ is a nonempty bounded subset of $\mathbb{R}^{n}$, then there exists a function $\phi:[0,+\infty) \rightarrow[0,+\infty]$ with $\phi(0)=0$ which is strictly increasing and such that

$$
\langle\xi-\zeta, u-v\rangle \geq \phi(\|u-v\|)
$$

whenever $u, v \in E$ and $\xi \in \partial f(u), \zeta \in \partial f(v)$. Observe that it is enough to prove that this condition is satisfied on sets $E$ which are closed balls of center zero. So, let $E$ be a closed ball of center zero and radius $M>0$. Denote

$$
E_{t}:=\left\{(u, v, \xi, \zeta) \in \mathbb{R}^{4 n}: u, v \in E,\|u-v\|=t, \xi \in \partial f(u), \zeta \in \partial f(v)\right\} .
$$

Define $\theta:[0,+\infty) \rightarrow[0,+\infty]$ by

$$
\theta(t)=\inf \left\{\langle\xi-\zeta, u-v\rangle:(u, v, \xi, \zeta) \in E_{t}\right\}
$$

Clearly, $\theta(t)=\infty$ for $t>2 M, \operatorname{dom} \theta=[0,2 M]$ and $\theta(0)=0$. Since $f$ is finite everywhere and convex, it is also continuous on $\mathbb{R}^{n}$ and, thus, it is bounded on bounded sets. Consequently, according to [28, Proposition 1.1.11], the multi-valued mapping $\partial f: \mathbb{R}^{n} \rightarrow \mathbb{R}^{n}$ transforms bounded sets into bounded sets. So, the set $E_{t}$ is contained in the bounded set $E \times E \times \partial f(E) \times \partial f(E)$. In order to show closedness of $E_{t}$, let $\left\{\left(u^{k}, v^{k}, \xi^{k}, \zeta^{k}\right)\right\}_{k \in \mathbb{N}}$ be a sequence contained in $E_{t}$ and converging to $(u, v, \xi, \zeta)$ in $\mathbb{R}^{4 n}$. Then, for each $k \in \mathbb{N}$, one has $\left\|u^{k}-v^{k}\right\|=t, \xi^{k} \in \partial f\left(u^{k}\right)$ and $\zeta^{k} \in \partial f\left(v^{k}\right)$. Since the mapping $\partial f$ is upper semicontinuous (cf. [57, Proposition 2.5]), it follows that $\xi \in \partial f(u)$ and $\zeta \in \partial f(v)$. Obviously, one also has that $\|u-v\|=t$ and these show that $(u, v, \xi, \zeta) \in E_{t}$, that is, $E_{t}$ is closed.

Due to the compactness of $E_{t}$, for any $t \in(0,2 M]$, there exists $u_{t}, v_{t} \in E$ and $\xi_{t} \in$ $\partial f\left(u_{t}\right), \zeta_{t} \in \partial f\left(v_{t}\right)$ such that

$$
\left\|u_{t}-v_{t}\right\|=t, \quad \theta(t)=\left\langle\xi_{t}-\zeta_{t}, u_{t}-v_{t}\right\rangle>0
$$

where the last inequality follows from the strict monotonicity of $\partial f$ because $f$ is strictly convex. We claim that $\theta$ is strictly increasing on $[0,2 M]$. To prove that, let $0<t_{1}<t_{2} \leq$ $2 M$. Denote $\bar{u}=u_{t_{2}}, \bar{v}=v_{t_{2}}, \bar{\xi}=\xi_{t_{2}}$ and $\bar{\zeta}=\zeta_{t_{2}}$. Let $\bar{t}=t_{1} / t_{2}$ and $\bar{w}:=\bar{u}+\bar{t}(\bar{v}-\bar{u})$. Clearly, $\bar{w} \in E$ and

$$
\|\bar{w}-\bar{u}\|=\bar{t}\|\bar{v}-\bar{u}\|=t_{1}
$$

Therefore, using the definition of $\theta$, one deduces that, for any $\eta \in \partial f(\bar{w})$, one has that

$$
\theta\left(t_{1}\right) \leq\langle\eta-\bar{\xi}, \bar{w}-\bar{u}\rangle=\bar{t}\langle\eta-\bar{\xi}, \bar{v}-\bar{u}\rangle \leq\langle\eta-\bar{\xi}, \bar{v}-\bar{u}\rangle .
$$

On the other hand, one has that

$$
\theta\left(t_{2}\right)=\langle\bar{\zeta}-\bar{\xi}, \bar{v}-\bar{u}\rangle=\langle\bar{\zeta}-\eta, \bar{v}-\bar{u}\rangle+\langle\eta-\bar{\xi}, \bar{v}-\bar{u}\rangle
$$


Since $\bar{\zeta} \in \partial f(\bar{v}), \eta \in \partial f(\bar{w})$ and $\partial f$ is strictly monotone (because $f$ is strictly convex), we deduce that $\langle\bar{\zeta}-\eta, \bar{v}-\bar{w}\rangle>0$. Thus, we have

$$
0<\langle\bar{\zeta}-\eta, \bar{v}-\bar{w}\rangle=(1-\bar{t})\langle\bar{\zeta}-\eta, \bar{v}-\bar{u}\rangle
$$

where $(1-\bar{t})>0$. This, (2.53) and (2.54) combined imply

$$
\theta\left(t_{2}\right)>\langle\eta-\bar{\xi}, \bar{v}-\bar{u}\rangle \geq \theta\left(t_{1}\right),
$$

proving that $\theta$ is strictly increasing on $[0,2 M]$.

Define the function $\phi:[0,+\infty) \rightarrow[0,+\infty]$ by

$$
\phi(s)= \begin{cases}2 \int_{0}^{s} \tau^{-1} \theta(\tau) d \tau & \text { if } s>0 \\ 0 & \text { if } s=0\end{cases}
$$

We are going to show that this function is well defined and satisfies condition (2.48). Let $t \in(0,2 M]$, let $u, v \in E$ be such that $\|u-v\|=t$ and let $\xi \in \partial f(u), \zeta \in \partial f(v)$. From the definition of $\theta$ one has that

$$
\langle\zeta-\xi, v-u\rangle \geq \theta(\|u-v\|)
$$

For any $s \in(0,1]$, denote $u_{s}:=u+s(v-u)$ and take $\eta_{s} \in \partial f\left(u_{s}\right)$. Then one gets

$$
\left\langle\eta_{s}-\xi, u_{s}-u\right\rangle \geq \theta\left(\left\|u_{s}-u\right\|\right)
$$

that is,

$$
\left\langle\eta_{s}-\xi, v-u\right\rangle \geq s^{-1} \theta(s\|v-u\|) .
$$

Letting $\tau=s\|v-u\|$ one obtains

$$
\frac{\left\langle\eta_{s}-\xi, v-u\right\rangle}{\|v-u\|} \geq \frac{\theta(\tau)}{\tau}
$$

Let $\left\{s_{k}\right\}_{k \in \mathbb{N}} \subset(0,1]$ be a sequence converging to zero. Then the sequence $\left\{u_{s_{k}}\right\}_{k \in \mathbb{N}}$ converges to $u$ and, due to the local boundedness of the operator $\partial f$, the sequence $\left\{\eta_{s_{k}}\right\}_{k \in \mathbb{N}}$ is bounded. So, by eventually taking a subsequence, we may assume that $\left\{\eta_{s_{k}}\right\}_{k \in \mathbb{N}}$ converges to some vector $\eta$. By the upper semicontinuity of the operator $\partial f$ we deduce that $\eta \in \partial f(u)$. Since in (2.61) the vector $\xi$ is an arbitrary element of $\partial f(u)$, it results that

$$
\frac{\left\langle\eta_{s_{k}}-\eta, v-u\right\rangle}{\|v-u\|} \geq \frac{\theta\left(\tau_{k}\right)}{\tau_{k}},
$$

where $\tau_{k}=s_{k}\|v-u\|$. So, letting $k \rightarrow \infty$ in (2.62), one deduces that $\lim _{\tau \rightarrow 0^{+}} \theta(\tau) / \tau=0$, and this implies that the function $\phi$ is well defined. Since $\theta$ is strictly increasing, it results that $\phi(s)=0$ if and only if $s=0$, and $\varphi$ is strictly increasing, too. From the inequality (2.60) one also has that

$$
\left\langle\eta_{s}, v-u\right\rangle \geq\langle\xi, v-u\rangle+s^{-1} \theta(s\|v-u\|) .
$$


If one chooses a point $\eta_{s} \in \partial f(u+s(v-u))$ for each $s \in[0,1]$, then the following integral exists and

$$
\int_{0}^{1}\left\langle\eta_{s}, v-u\right\rangle d s=f(v)-f(u) .
$$

This fact was proved in a particular case in [30] and the proof can be extended without much change. Hence, integrating in (2.63), one gets

$$
f(v)-f(u) \geq\langle\xi, v-u\rangle+\int_{0}^{1} s^{-1} \theta(s\|v-u\|) d s .
$$

Interchanging $u$ and $v$ in the previous arguments one deduces that

$$
f(u)-f(v) \geq\langle\zeta, u-v\rangle+\int_{0}^{1} s^{-1} \theta(s\|v-u\|) d s .
$$

Adding the last two inequalities one obtains

$$
\langle\zeta-\xi, v-u\rangle \geq 2 \int_{0}^{1} s^{-1} \theta(s\|v-u\|) d s=2 \int_{0}^{\|v-u\|} \frac{\theta(\tau)}{\tau} d \tau=\phi(\|v-u\|) .
$$

This completes the proof.

\section{Totally convex Banach spaces}

3.1. Identifying totally convex functions in Banach spaces of infinite dimension is a problem of practical interest in optimization and in other areas. If they happen to be totally convex, then the functions $h_{r}=\|\cdot\|^{r}, r>1$, are among the most convenient such functions to work with (see [28, Chapter 3], [7]). Thus, it is natural to ask whether, and under which conditions, the function $h_{r}$ is totally convex as to ensure that $D$-convergence and norm convergence are equivalent. Recall that, in uniformly convex Banach spaces, uniform convexity of the function $h_{r}$ and then, implicitly, total convexity, was established by Asplund [10], Clarkson [38], Vladimirov et al. [67] for $r \geq 2$. Zălinescu [68] proved that for $r>1$, the functions $h_{r}$ are uniformly convex at any $x \in X$ in locally uniformly convex spaces, and thus, totally convex at any $x \in X$. This also follows from the next result which gives evaluations of the moduli of total convexity of $h_{r}$ in (locally) uniformly convex Banach spaces. These evaluations were established in $[30,31]$ and were used in the design and convergence analysis of the algorithms presented in [28, Chapter 3], [8, 33].

Proposition 3.1. (i) If $X$ is a locally uniformly convex Banach space and $\phi(t):=|t|^{r}$, then, for any $t \geq 0$, one has

$$
\mu_{h_{r}}(0, t)=\left(1-2^{1-r}\right) t^{r}
$$

and, when $x \neq 0$ and $\alpha$ is any number in $(0,1)$, one has

$$
v_{h_{r}}(x, t) \geq \mu_{h_{r}}(x, t) \geq\|x\|^{r} \min \left\{\mu_{\phi}\left(1, \frac{\alpha t}{\|x\|}\right), \zeta(a)\right\}
$$


where $\zeta:[0,1] \rightarrow[0, \infty)$ is the function

$$
\begin{gathered}
\zeta(s)= \begin{cases}1-\left[2-(1-2 s)^{r /(r-1)}\right]^{1-r} & \text { if } s \in\left[0, \frac{1}{2}\right), \\
1-2^{1-r} & \text { if } s \geq \frac{1}{2},\end{cases} \\
\mu_{\phi}(1, s)=\min \left\{1+(1+s)^{r}-2^{1-r}(2+s)^{r}, 1+|1-s|^{r}-2^{1-r}|2-s|^{r}\right\}, \\
a:=\delta_{X}\left(\frac{x}{\|x\|},(1-\alpha) \frac{t}{\|x\|}\right) ;
\end{gathered}
$$

(ii) If $X$ is uniformly convex and $r \in(1, \infty)$, then the function $h_{r}$ is totally convex, and, for any $z \in X$ and $t>0$, one has:

$$
\nu_{h_{r}}(z, t) \geq r M\left(\frac{t}{2}\right)^{r} \int_{0}^{1} \tau^{r-1} \delta_{X}\left(\frac{\tau t}{2(\|z\|+\tau t)}\right) d \tau>0
$$

where $M$ is some positive constant and $\delta_{X}$ is the modulus of convexity of $X$.

3.2. It is known that geometric properties of Banach spaces, such as strict convexity, locally uniform convexity, uniform convexity can be characterized analytically by properties of the square of the norm (see, for instance, [37]). In [62], a Banach space $X$ is called locally totally convex if the function $h=(1 / 2)\|\cdot\|^{2}$ is totally convex at each $x \in X$.

Locally uniformly convex spaces are locally totally convex; separable or reflexive Banach spaces can be equivalently renormed for becoming locally uniformly convex spaces (see $[50,66]$, resp.).

It follows from [69, Section 3.7] that (locally) uniform convexity of a Banach space is equivalent to (locally) uniform convexity of a large class of functions $\Phi$ of the norm, where

$$
\Phi(x)=\int_{0}^{\|x\|} \varphi(s) d s
$$

and $\varphi$ is a weight function. An analogous result is true in the case of total convexity, as shown by the result below. Recall that the Banach space $X$ is said to be an E-space if it is reflexive, strictly convex and has the Kadec-Klee property. According to [37, page 47], a Banach space is an E-space if and only if it is weakly uniformly convex, that is, for any two sequences $\left\{x^{n}\right\}_{n \in \mathbb{N}},\left\{y^{n}\right\}_{n \in \mathbb{N}} \subseteq X$ with $\left\|x^{n}\right\|=\left\|y^{n}\right\|=1, n \in \mathbb{N}$, one has

$$
\lim _{n \rightarrow \infty}\left\langle x^{*}, x^{n}+y^{n}\right\rangle=2 \Longrightarrow \lim _{n \rightarrow \infty}\left\|x^{n}-y^{n}\right\|=0,
$$

whenever $x^{*} \in X^{*},\left\|x^{*}\right\|=1$. The E-spaces, introduced by Fan and Glicksberg [44], were studied mostly because they are the natural setting for the research of strong Tykhonov and Hadamard well-posedness of convex best approximation problems (see, e.g., [39, 46]), and variational inequalities in Banach spaces (see, e.g., [7]).

Proposition 3.2 (cf. [62]). The following conditions are equivalent:

(i) $X$ is locally totally convex; 
18 Bregman distances, total convexity, operator equations

(ii) there exists a weight function $\varphi$ such that the function $\Phi$ defined at (3.5), is totally convex at any $x \in X$;

(iii) for any weight function $\varphi$, the function $\Phi$ is totally convex at any $x \in X$;

(iv) the function $(1 / 2)\|\cdot\|^{2}$ is totally convex at each point of the unit sphere of $X$. Moreover, if $X$ is reflexive, then these conditions are also equivalent to the following:

(v) $X$ is an E-space;

(vi) X has the Kadec-Klee property.

3.3. The next result shows another characterization of locally totally convex spaces and points out some geometric properties of the norm in this context. It was suggested to us by Constantin Zălinescu. In view of Proposition 3.2, if $X$ is reflexive, then this result provides also a characterization of the $E$-spaces. In what follows, we denote by $S_{X}$ the unit sphere of $X$.

Theorem 3.3. Let $X$ be a Banach space. Then the following conditions are equivalent:

(i) the space $X$ is locally totally convex;

(ii) for any $x \in S_{X}$ and for any real number $\varepsilon>0$, there exists $\delta=\delta(x, \varepsilon)>0$ such that, for all $y \in S_{X}$ with $\|y-x\| \geq \varepsilon$, there exists $\lambda_{0} \in(0,1)$ such that

$$
\left\|\left(1-\lambda_{0}\right) x+\lambda_{0} y\right\|<1-\lambda_{0} \delta
$$

(iii) for any $x \in S_{X}$ and for any real number $\varepsilon>0$, there exists $\delta=\delta(x, \varepsilon)>0$ such that, for all $y \in S_{X}$ with $\|y-x\|=\varepsilon$, there exists $\lambda_{0} \in(0,1)$ such that the inequality (3.7) holds.

Proof. (i) $\Rightarrow$ (ii). Let $h=(1 / 2)\|\cdot\|^{2}$. Suppose, by contradiction, that for some $\left\|x^{0}\right\|=1$, for some $\varepsilon_{0}>0$ and for any $n \in \mathbb{N}$, there is $y^{n} \in X$ with $\left\|y^{n}\right\|=1$ and $\left\|y^{n}-x^{0}\right\| \geq \varepsilon_{0}$ such that

$$
\left\|x^{0}+\lambda\left(y^{n}-x^{0}\right)\right\| \geq 1-\frac{\lambda}{n}=\left\|x^{0}\right\|-\frac{\lambda}{n}
$$

whenever $\lambda \in(0,1)$. Subtracting $\left\|x^{0}\right\|$, dividing the resulting inequality by $\lambda$ and letting $\lambda \rightarrow 0^{+}$, one gets $-g^{\circ}\left(x^{0}, y^{n}-x^{0}\right) \leq 1 / n$, where $g=\|\cdot\|$. Consequently, $D_{h}\left(y^{n}, x^{0}\right) \leq 1 / n$ for any $n \in \mathbb{N}$, since $h^{\circ}\left(x^{0}, y^{n}-x^{0}\right)=\left\|x^{0}\right\| \cdot g^{\circ}\left(x^{0}, y^{n}-x^{0}\right)$. By Proposition 2.5 and the total convexity of $h$ at $x^{0}$, it follows that $\left\{y^{n}\right\}_{n \in \mathbb{N}}$ converges to $x^{0}$, which is a contradiction.

(ii) $\Rightarrow($ i). According to Proposition 2.1(v) and Proposition 3.2, it is sufficient to show that

$$
\inf \left\{D_{h}(y, x):\|y-x\| \geq \varepsilon\right\}>0
$$

for any $x \in S_{X}$ and $\varepsilon>0$. Take $y \in X$ with $\|y-x\| \geq \varepsilon$. Denote $v=y /\|y\|$ and $\varepsilon_{0}=\varepsilon / 2$. Then, for some $\delta=\delta(x, \varepsilon)>0$ and for any $w \in S_{X}$ with $\|x-w\| \geq \varepsilon_{0}$, there is $\lambda_{0} \in(0,1)$ such that

$$
1-\left\|x+\lambda_{0}(w-x)\right\|>\lambda_{0} \delta
$$


Observe that

$$
D_{h}(y, x) \geq \frac{1}{2}\|y\|^{2}-\frac{1}{2}-\frac{\|x+\lambda(y-x)\|^{2}-1}{2 \lambda}:=\zeta_{\lambda}(x, y)
$$

for any $\lambda \in(0,1)$, because

$$
h^{\circ}(x, y-x) \leq \frac{h(x+\lambda(y-x))-h(x)}{\lambda} .
$$

We distinguish the following possible situations:

Case 1. If $\|x\|-\|y\| \geq \varepsilon / 2$, then

$$
\zeta_{1 / 2}(x, y) \geq \frac{1}{4}(\|x\|-\|y\|)^{2} \geq \frac{1}{16} \varepsilon^{2} .
$$

By using (3.11) and taking infimum over $y \in X$ such that $\|y-x\| \geq \varepsilon$, inequality (3.9) follows.

Case 2. Suppose that $0 \leq\|x\|-\|y\|<\varepsilon / 2$. Denote $\alpha=1 /\|y\|, \beta=\left(\lambda_{0}+\alpha^{2}\left(1-\lambda_{0}\right)\right)^{1 / 2}$, $z=x+\lambda_{0}(v-x)$, and $\gamma=\|z\|+\left(1-\lambda_{0}\right)(\alpha-1)$. Then one has

$$
x+\lambda_{0}(y-x)=\|y\|\left[z+\left(1-\lambda_{0}\right)(\alpha-1) x\right] .
$$

Note that

$$
\left\|x+\lambda_{0}(y-x)\right\| \leq\|y\|\left[\|z\|+\left(1-\lambda_{0}\right)(\alpha-1)\right]
$$

Then one gets

$$
\begin{aligned}
D_{h}(y, x) \geq \zeta_{\lambda_{0}}(x, y) & \geq \frac{\|y\|^{2}}{2}\left(1-\alpha^{2}-\frac{\gamma^{2}-\alpha^{2}}{\lambda_{0}}\right) \\
& =\frac{\|y\|^{2}}{2 \lambda_{0}}(\beta+\gamma)(\beta-\gamma) \geq \frac{\|y\|^{2}}{2 \lambda_{0}}(1-\|z\|),
\end{aligned}
$$

where the last inequality holds because $\beta+\gamma \geq 1$ and $\beta \geq 1+\left(1-\lambda_{0}\right)(\alpha-1)$. Observe that

$$
\|v-x\|=\left\|x-y+\frac{\|y\|-1}{\|y\|} y\right\| \geq[\|x-y\|-(1-\|y\|)] \geq \frac{\varepsilon}{2}=\varepsilon_{0} .
$$

If $\|y\| \geq \varepsilon / 2$ then, by using (3.10), one obtains that $\zeta_{\lambda_{0}}(x, y) \geq \delta \varepsilon^{2} / 8$. Otherwise, one has $\|x\|>\varepsilon / 2$ because $\|y-x\| \geq \varepsilon$. Consequently, $\|y\|>\|x\|-\varepsilon / 2>0$ and, thus,

$$
\zeta_{\lambda_{0}}(x, y) \geq \frac{\delta}{2}\left(\|x\|-\frac{\varepsilon}{2}\right)^{2}>0 .
$$

Therefore, in both situations, inequality (3.9) holds.

Case 3. Suppose that $0 \leq\|y\|-\|x\|<\varepsilon / 2$. If one denotes $\alpha=\|y\|$ and preserves the notations for $\beta$ and $\gamma$, then the argument in this case is similar to that in Case 2. 
Case 4. If $\|y\|-\|x\| \geq \varepsilon / 2$, then one proceeds as in Case 1 with $x$ and $y$ interchanged.

The proof of (i) $\Leftrightarrow$ (iii) is identical to that of (i) $\Leftrightarrow($ ii), due to Proposition 2.1(v).

3.4. As pointed out in Section 2.9, there are totally convex functions which are not locally uniformly convex. However, we do not know any example of a Banach space which is locally totally convex without being locally uniformly convex. The following proposition seems to support our conjecture that locally totally convex spaces are not necessarily locally uniformly convex. The key fact is that $\lambda_{0}$ in the statement below does not depend on $y$, while in the previous theorem it does.

Proposition 3.4. Let X be a Banach space. Then the following conditions are equivalent:

(i) the space $X$ is locally uniformly convex;

(ii) for any $x \in S_{X}$ and for any real number $\varepsilon>0$, there exists $\delta=\delta(x, \varepsilon)>0$ and $\lambda_{0} \in$ $(0,1)$ such that, for all $y \in S_{X}$ with $\|y-x\| \geq \varepsilon$, one has

$$
\left\|\left(1-\lambda_{0}\right) x+\lambda_{0} y\right\|<1-\lambda_{0} \delta
$$

Proof. (i) $\Rightarrow$ (ii). Let $x \in S_{X}$ and $\varepsilon>0$. From the definition of locally uniformly convex spaces, there exists $\delta>0$ and $\lambda_{0}=1 / 2$ such that (3.19) holds.

(ii) $\Rightarrow$ (i). It follows immediately.

\section{Relative projections onto closed convex sets}

4.1. In this section we present the basic properties of a class of operators we call relative projections. They are natural generalizations of the Bregman projections introduced in [20] and of the generalized projections defined and studied by Alber [3, 5]. In the sequel we assume that the Banach space $X$ is reflexive and $f: X \rightarrow(-\infty,+\infty]$ represents a proper lower semicontinuous function which is strictly convex on its domain $\operatorname{dom} f$. As usual, we denote by $f^{*}: X^{*} \rightarrow(-\infty,+\infty]$ the Fenchel conjugate of $f$, that is, the function defined by

$$
f^{*}(\xi):=\sup \{\langle\xi, x\rangle-f(x): x \in X\} .
$$

We follow Alber [5] and, with the function $f$ we associate the function $W^{f}: X^{*} \times X \rightarrow$ $(-\infty,+\infty]$ defined by

$$
W^{f}(\xi, x)=f(x)-\langle\xi, x\rangle+f^{*}(\xi)
$$

Clearly, $W^{f}$ is nonnegative and, for any $\xi \in \operatorname{dom} f^{*}$, the function $W^{f}(\xi, \cdot)$ is strictly convex.

4.2. For any $\xi \in X^{*}$ and for any subset $K$ of $X$ denote

$$
W^{f}(\xi, K)=\inf \left\{W^{f}(\xi, x): x \in K\right\}
$$


An element $y \in K$ such that

$$
W^{f}(\xi, y)=W^{f}(\xi, K)
$$

is called projection of $\xi$ onto $K$ relative to $f$. The following result shows sufficient conditions for existence of such projections. In the case of a strongly coercive function $f$, this result, as well as Proposition 4.3 below occur in [5].

Proposition 4.1. Suppose that $\xi \in \operatorname{dom} f^{*}$ and that the set $K \subseteq \operatorname{dom} f$ is nonempty, closed and convex. If the set

$$
R_{\alpha}^{f}(\xi)=\left\{z \in \operatorname{dom} f: W^{f}(\xi, z) \leq \alpha\right\}
$$

is bounded for any $\alpha \in[0,+\infty)$, then there exists a unique projection of $\xi$ onto $K$ relative to $f$. In particular, this happens if any of the following conditions holds:

(i) $\xi \in \operatorname{range}(\partial f)$ and $f$ is totally convex at each point of its domain;

(ii) $f$ is strongly coercive (in the sense that $\lim _{\|x\| \rightarrow \infty}(f(x) /\|x\|)=+\infty$ ).

Proof. It was noted in Section 4.1 that the function $W^{f}(\xi, \cdot)$ is strictly convex. This guarantees that there is no more than one vector $y$ satisfying (4.4). Since the function $W^{f}(\xi, \cdot)$ is also convex, lower semicontinuous and $R_{\alpha}^{f}(\xi)$ is bounded for each $\alpha \in[0,+\infty)$, it results that $W^{f}(\xi, \cdot)$ has at least one minimizer in the convex set $K$, that is, the projection of $\xi$ onto $K$ relative to $f$ exists.

Suppose that $\xi \in \operatorname{range}(\partial f), f$ is totally convex and that, for some $\alpha \geq 0$, the set $R_{\alpha}^{f}(\xi)$ is unbounded. Then there exists a sequence $\left\{y^{k}\right\}_{k \in \mathbb{N}}$ contained in $R_{\alpha}^{f}(\xi)$ such that $\lim _{k \rightarrow \infty}\left\|y^{k}\right\|=+\infty$. According to Proposition 2.1, for any $x \in \operatorname{dom} f$ and for any natural number $k$ such that $\left\|y^{k}-x\right\| \geq 1$, one has

$$
\begin{aligned}
\alpha \geq W^{f}\left(\xi, y^{k}\right) & =D_{f}\left(y^{k}, x\right)+f^{\circ}\left(x, y^{k}-x\right)-\left\langle\xi, y^{k}-x\right\rangle+W^{f}(\xi, x) \\
& \geq v_{f}(x, 1)\left\|y^{k}-x\right\|+f^{\circ}\left(x, y^{k}-x\right)-\left\langle\xi, y^{k}-x\right\rangle .
\end{aligned}
$$

Since $\xi \in \operatorname{range}(\partial f)$, there exists a vector $x^{0} \in \operatorname{dom} \partial f$ such that $\xi \in \partial f\left(x^{0}\right)$. One can check (or see, e.g., [57, page 41]) that

$$
f^{\circ}\left(x^{0}, y^{k}-x^{0}\right) \geq\left\langle\xi, y^{k}-x^{0}\right\rangle .
$$

This, combined with (4.6), implies that

$$
\alpha \geq v_{f}\left(x^{0}, 1\right)\left\|y^{k}-x^{0}\right\| .
$$

Since $f$ is totally convex at $x^{0}$, it results that $\nu_{f}\left(x^{0}, 1\right)>0$. Therefore, by letting $k \rightarrow \infty$ in (4.8), one gets a contradiction. Hence, the set $R_{\alpha}^{f}(\xi)$ is bounded for all $\alpha \geq 0$.

Now, suppose that $f$ is strongly coercive and that, for some $\alpha \geq 0$, there exists a sequence $\left\{x^{k}\right\}_{k \in \mathbb{N}}$ contained in $R_{\alpha}^{f}(\xi)$ such that $\lim _{k \rightarrow \infty}\left\|x^{k}\right\|=+\infty$. Then

$$
\alpha \geq W^{f}\left(\xi, x^{k}\right) \geq f^{*}(\xi)+\left\|x^{k}\right\|\left(\frac{f\left(x^{k}\right)}{\left\|x^{k}\right\|}-\|\xi\|_{*}\right)
$$


and letting here $k \rightarrow+\infty$ one gets a contradiction. Hence, in this case too, the set $R_{\alpha}^{f}(\xi)$ is bounded for all $\alpha \geq 0$.

4.3. Whenever it exists, we denote by $P_{K}^{f}(\xi)$ the projection of $\xi$ onto $K \subseteq \operatorname{dom} f$ with respect to $f$. The function $\xi \rightarrow P_{K}^{f}(\xi): \operatorname{dom} P_{K}^{f} \rightarrow K$ is called the projection operator onto $K$ relative to $f$. Proposition 4.1 shows that if $f$ is totally convex, then $\operatorname{range}(\partial f) \subseteq \operatorname{dom} P_{K}^{f}$. Also, if $f$ is strongly coercive, then $\operatorname{dom} P_{K}^{f}=\operatorname{dom} f^{*}$. One can ask whether strongly coercivity implies total convexity or vice-versa. The examples below show that this is not the case. First, consider the situation when $X=\mathbb{R}$ and $f: \mathbb{R} \rightarrow[0,+\infty)$ is given by $f(x)=e^{-x}$. This function is not strongly coercive, while, by Proposition 2.2 , it is totally convex at any point of its domain. Also, it was noted in Section 2.14 that there are Banach spaces on which the strongly coercive function $(1 / 2)\|\cdot\|^{2}$ is strictly convex (even Gâteaux differentiable-see [15]) without being totally convex at all points of the space.

Note that the existence of relative projections onto closed convex sets does not involve differentiability requirements on $f$. However, in applications, the case when $f$ is Gâteaux differentiable is of most interest. From now on, and until the end of this section, we presume that the following condition holds.

Assumption 4.2. The function $f$ is Gâteaux differentiable on $\operatorname{int}(\operatorname{dom} f)$.

In this situation, one has that

$$
f^{*}\left(f^{\prime}(x)\right)=\left\langle f^{\prime}(x), x\right\rangle-f(x),
$$

for any $x \in \operatorname{int}(\operatorname{dom} f)$ and, consequently,

$$
D_{f}(y, x)=W^{f}\left(f^{\prime}(x), y\right) .
$$

Recall that the Bregman projection of $x$ onto the nonempty closed convex set $K \subseteq \operatorname{dom} f$ (as defined in [20]), is the necessarily unique vector $\Pi_{K}^{f}(x) \in K$, satisfying

$$
D_{f}\left(\Pi_{K}^{f}(x), x\right)=\inf \left\{D_{f}(y, x): y \in K\right\} .
$$

According to Proposition 4.1, since $f^{\prime}(x) \in \operatorname{int}\left(\operatorname{dom} f^{*}\right)$ for all $x \in \operatorname{int}(\operatorname{dom} f)$, one has that

$$
\Pi_{K}^{f}(x)=P_{K}^{f}\left(f^{\prime}(x)\right)=\left(P_{K}^{f} \circ f^{\prime}\right)(x) .
$$

4.4. Similarly to the metric projection in Hilbert spaces, Bregman projections and generalized projections (i.e., the projections relative to $h=(1 / 2)\|\cdot\|^{2}$ ) have variational characterizations-see $[3,6]$. This property extends to relative projections with respect to totally convex differentiable functions.

Proposition 4.3. Let $f$ be totally convex at any point of $\operatorname{int}(\operatorname{dom} f)$ and let $K \subseteq \operatorname{int}(\operatorname{dom}$ $f$ ) be a nonempty, closed and convex set. If $\xi \in X^{*}$ and $\hat{x} \in K$, then the following conditions are equivalent:

(a) The vector $\hat{x}$ is the projection of $\xi$ onto $K$ relative to $f$; 
(b) The vector $\hat{x}$ is the unique solution in $K$ of the following variational inequality:

$$
\left\langle\xi-f^{\prime}(z), z-y\right\rangle \geq 0, \quad \forall y \in K
$$

(c) The vector $\hat{x}$ is the unique solution contained in $K$ of the following variational inequality:

$$
W^{f}(\xi, z)+W^{f}\left(f^{\prime}(z), y\right) \leq W^{f}(\xi, y), \quad \forall y \in K
$$

Proof. We prove first that (a) and (b) are equivalent. Suppose that (a) holds. Then, for any $x \in K$ one has $W^{f}(\xi, \hat{x}) \leq W^{f}(\xi, x)$. In particular, this holds for $x=(1-\tau) \hat{x}+\tau y$ for all $y \in K$ and for all $\tau \in[0,1]$. Hence, one obtains

$$
0 \geq W^{f}(\xi, \hat{x})-W^{f}(\xi,(1-\tau) \hat{x}+\tau y) \geq\left\langle\left[W^{f}(\xi, \cdot)\right]^{\prime}((1-\tau) \hat{x}+\tau y), \tau(\hat{x}-y)\right\rangle,
$$

where $\left[W^{f}(\xi, \cdot)\right]^{\prime}=f^{\prime}-\xi$. Therefore, for any $\tau \in(0,1]$, one has that

$$
0 \geq\left\langle f^{\prime}((1-\tau) \hat{x}+\tau y)-\xi, \hat{x}-y\right\rangle
$$

and, letting here $\tau \rightarrow 0^{+}$, one obtains (4.14) because the function $\left\langle f^{\prime}(\cdot)-\xi, \hat{x}-y\right\rangle$ is continuous due to the norm-to-weak ${ }^{*}$ continuity of $f^{\prime}$. Now, suppose that $\hat{x} \in K$ satisfies (4.14). Then, for any $y \in K$, one has

$$
W^{f}(\xi, y)-W^{f}(\xi, \hat{x}) \geq\left\langle\left[W^{f}(\xi, \cdot)\right]^{\prime}(\hat{x}), y-\hat{x}\right\rangle=\left\langle f^{\prime}(\hat{x})-\xi, y-\hat{x}\right\rangle \geq 0,
$$

showing that $\hat{x}$ minimizes $W^{f}(\xi, \cdot)$ over $K$, that is, $\hat{x}=P_{K}^{f}(\xi)$. In order to show that (b) and (c) are equivalent, it is sufficient to observe that

$$
W^{f}(\xi, \hat{x})+W^{f}\left(f^{\prime}(\hat{x}), y\right)-W^{f}(\xi, y)=\left\langle f^{\prime}(\hat{x})-\xi, \hat{x}-y\right\rangle
$$

for any $y \in K$.

4.5. Combining Proposition 4.3 with (4.13), one re-finds the variational characterizations of the Bregman projections given in [28].

Corollary 4.4. Suppose that $f$ is totally convex at any point of $\operatorname{int}(\operatorname{dom} f)$. Let $x \in$ $\operatorname{int}(\operatorname{dom} f)$ and let $K \subseteq \operatorname{int}(\operatorname{dom} f)$ be a nonempty, closed and convex set. If $\hat{x} \in K$, then the following conditions are equivalent:

(i) the vector $\hat{x}$ is the Bregman projection of $x$ onto $K$ with respect to $f$;

(ii) the vector $\hat{x}$ is the unique solution of the variational inequality

$$
\left\langle f^{\prime}(x)-f^{\prime}(z), z-y\right\rangle \geq 0, \quad \forall y \in K
$$

(iii) the vector $\hat{x}$ is the unique solution of the variational inequality

$$
D_{f}(y, z)+D_{f}(z, x) \leq D_{f}(y, x), \quad \forall y \in K .
$$


4.6. If $X$ is a Hilbert space and $f=(1 / 2)\|\cdot\|^{2}$, then the operators $P_{K}^{f}$ and $\Pi_{K}^{f}$ coincide and are equal to the metric projection operator onto the set $K$. It is known (see, for instance, [39]) that the metric projection operators in Hilbert spaces have valuable continuity and stability properties. Such properties were also proved in [61] for Bregman projections with respect to totally convex functions. As shown by the next result, which extends [61, Theorem 2.2.12] and [47, Theorem 4.1], some of these properties remain true for the relative projection operators $P_{K}^{f}$ in any reflexive Banach space. Recall (cf. [54]) that a sequence $\left\{K_{n}\right\}_{n \in \mathbb{N}}$ of subsets of $X$ is called convergent (in Mosco's sense) to the set $K$ (we write $M$-convergent for short) if

$$
K=w-\varlimsup_{n \rightarrow \infty} K_{n}=s-\underline{\lim }_{n \rightarrow \infty} K_{n}
$$

where $s-\underline{\lim }_{n \rightarrow \infty} K_{n}$ represents the collection of all $y \in X$ which are limits (in the strong convergence sense) of sequences $\left\{x^{k}\right\}_{k \in \mathbb{N}}$ with the property that $x^{n} \in K_{n}$ for all $n \in \mathbb{N}$ and $w-\overline{\lim }_{n \rightarrow \infty} K_{n}$ denotes the collection of all $x \in X$ such that there exists a sequence $\left\{y^{n}\right\}_{n \in \mathbb{N}}$ in $X$ converging weakly to $x$ and with the property that there exists a subsequence $\left\{K_{i_{n}}\right\}_{n \in \mathbb{N}}$ of $\left\{K_{n}\right\}_{n \in \mathbb{N}}$ such that $y^{n} \in K_{i_{n}}$ for all $n \in \mathbb{N}$.

Theorem 4.5. Suppose that $f$ is lower semicontinuous and at least one of the following conditions is satisfied:

(i) $f$ is strongly coercive;

(ii) $f$ is totally convex on bounded sets.

If $K$ and $K_{n},(n \in \mathbb{N})$, are nonempty closed convex subsets of $\operatorname{int}(\operatorname{dom} f)$ and if the sequence $\left\{K_{n}\right\}_{n \in \mathbb{N}}$ is $M$-convergent to $K$, then for any sequence $\left\{\xi^{n}\right\}_{n \in \mathbb{N}}$ contained in int(dom $\left.f^{*}\right)$ which converges to some $\xi \in \operatorname{int}\left(\operatorname{dom} f^{*}\right)$, the following limits exist and we have

$$
\begin{gathered}
\lim _{n \rightarrow \infty} W^{f}\left(\xi^{n}, K_{n}\right)=W^{f}(\xi, K), \\
w-\lim _{n \rightarrow \infty} P_{K_{n}}^{f}\left(\xi^{n}\right)=P_{K}^{f}(\xi) .
\end{gathered}
$$

Proof. Denote $\hat{x}^{n}=P_{K_{n}}^{f}\left(\xi^{n}\right)$ and $\hat{x}=P_{K}^{f}(\xi)$. Suppose that $\left\{z^{n}\right\}_{n \in \mathbb{N}}$ is a sequence in $X$ such that $z^{n} \in K_{n}$ for all $n \in \mathbb{N}$ and $\lim _{n \rightarrow \infty} z^{n}=z$. Since $\left\{K_{n}\right\}_{n \in \mathbb{N}}$ is $M$-convergent to $K$ it results that $z \in K$. By Proposition 4.3 one deduces that

$$
W^{f}\left(\xi^{n}, \hat{x}^{n}\right)+W^{f}\left(f^{\prime}\left(\hat{x}^{n}\right), z^{n}\right) \leq W^{f}\left(\xi^{n}, z^{n}\right), \quad \forall n \in \mathbb{N} .
$$

Since $f$ and $f^{*}$ are lower semicontinuous they are continuous on the interior of their respective domains. Consequently, $W^{f}$ is continuous on $\operatorname{int}\left(\operatorname{dom} f^{*}\right) \times \operatorname{int}(\operatorname{dom} f)$ and, therefore, the sequence $\left\{W^{f}\left(\xi^{n}, z^{n}\right)\right\}_{n \in \mathbb{N}}$ is convergent. By (4.25), this implies that the sequence $\left\{W^{f}\left(\xi^{n}, \hat{x}^{n}\right)\right\}_{n \in \mathbb{N}}$ is bounded. We claim that $\left\{\hat{x}^{n}\right\}_{n \in \mathbb{N}}$ is bounded too. In order to show that, suppose first that $f$ is coercive. Then the following inequality, which results immediately from the definition of $W^{f}\left(\xi^{n}, \hat{x}^{n}\right)$, cannot hold unless $\left\{\hat{x}^{n}\right\}_{n \in \mathbb{N}}$ is bounded:

$$
W^{f}\left(\xi^{n}, \hat{x}^{n}\right) \geq f^{*}\left(\xi^{n}\right)+\left\|\hat{x}^{n}\right\|\left(\frac{f\left(\hat{x}^{n}\right)}{\left\|\hat{x}^{n}\right\|}-\left\|\xi^{n}\right\|\right) .
$$


Now, assume that $f$ is totally convex on bounded sets. By Theorem 2.10, the function $f$ is also uniformly convex on bounded sets. Thus, according to [69, Proposition 2.4.13], for each $n \in \mathbb{N}$, one has that

$$
\xi^{n} \in \operatorname{int}\left(\operatorname{dom} f^{*}\right)=\operatorname{int}\left(\operatorname{dom} \partial f^{*}\right)=\operatorname{int}(\operatorname{range}(\partial f))=\operatorname{int}\left(\operatorname{range}\left(f^{\prime}\right)\right) .
$$

Thus, for each $n \in \mathbb{N}$, there exists $u^{n} \in \operatorname{int}(\operatorname{dom} f)$ such that $f^{\prime}\left(u^{n}\right)=\xi^{n}$. The sequence $\left\{u^{n}\right\}_{n \in \mathbb{N}}$ is contained in the set $\bigcup_{n \in \mathbb{N}} \partial f^{*}\left(\xi^{n}\right)$. Since the operator $\partial f^{*}$ is monotone, it is also locally bounded and, so, there exists a neighborhood $V$ of $\xi$ such that $\bigcup_{\zeta \in V} \partial f^{*}(\zeta)$ is bounded. The sequence $\left\{\xi^{n}\right\}_{n \in \mathbb{N}}$ being convergent to $\xi$, there exists a positive integer $n_{0}$ such that $\xi^{n} \in V$ for all $n \geq n_{0}$. Hence, for any $n \geq n_{0}$ one has

$$
u^{n} \in \partial f^{*}\left(\xi^{n}\right) \subseteq \bigcup_{\zeta \in V} \partial f^{*}(\zeta)
$$

and this implies that the sequence $\left\{u^{n}\right\}_{n \in \mathbb{N}}$ is bounded. Thus, the function $v_{f}\left(\left\{u^{n}\right\}_{n \in \mathbb{N}}, \cdot\right)$ is strictly increasing (see Section 2.10). Observe that

$$
v_{f}\left(\left\{u^{n}\right\}_{n \in \mathbb{N}},\left\|\hat{x}^{n}-u^{n}\right\|\right) \leq D_{f}\left(\hat{x}^{n}, u^{n}\right)=W^{f}\left(\xi^{n}, \hat{x}^{n}\right),
$$

where the sequence $\left\{W^{f}\left(\xi^{n}, \hat{x}^{n}\right)\right\}_{n \in \mathbb{N}}$ is bounded. Since the sequence $\left\{u^{n}\right\}_{n \in \mathbb{N}}$ is also bounded, this inequality cannot hold unless the sequence $\left\{\hat{x}^{n}\right\}_{n \in \mathbb{N}}$ is bounded too.

We show next that the only weak accumulation point of $\left\{\hat{x}^{n}\right\}_{n \in \mathbb{N}}$ is $\hat{x}$. Clearly, this implies (4.24). To this end, note that, if $\left\{\zeta^{n}\right\}_{n \in \mathbb{N}}$ is strongly convergent to $\zeta$ in $X^{*}$ and if $\left\{u^{n}\right\}_{n \in \mathbb{N}}$ is weakly convergent to $u$ in $X$, then

$$
W^{f}(\zeta, u) \leq \liminf _{n \rightarrow \infty} W^{f}\left(\zeta^{n}, u^{n}\right)
$$

because of the weak lower semicontinuity of $f$ and the lower semicontinuity of $f^{*}$. Since $\hat{x} \in K$ and $K$ is the Mosco limit of the sequence of sets $\left\{K_{n}\right\}_{n \in \mathbb{N}}$, there exists a sequence $\left\{v^{n}\right\}_{n \in \mathbb{N}}$ in $X$ such that $v^{n} \in K_{n}$ for all $n \in \mathbb{N}$ and $\lim _{n \rightarrow \infty} v^{n}=\hat{x}$. Let $\bar{x}$ be an arbitrary weak accumulation point of $\left\{\hat{x}^{n}\right\}_{n \in \mathbb{N}}$ and let $\left\{\hat{x}^{i_{n}}\right\}_{n \in \mathbb{N}}$ be a subsequence of $\left\{\hat{x}^{n}\right\}_{n \in \mathbb{N}}$ which converges weakly to $\bar{x}$. Clearly, $\bar{x} \in K$ and, by (4.30), one has

$$
\begin{aligned}
W^{f}(\xi, \hat{x}) & \leq W^{f}(\xi, \bar{x}) \leq \liminf _{n \rightarrow \infty} W^{f}\left(\xi^{i_{n}}, \hat{x}^{i_{n}}\right) \\
& \leq \liminf _{n \rightarrow \infty} W^{f}\left(\xi^{i_{n}}, v^{i_{n}}\right)=\lim _{n \rightarrow \infty} W^{f}\left(\xi^{n}, v^{n}\right)=W^{f}(\xi, \hat{x}) .
\end{aligned}
$$

This shows that $W^{f}(\xi, \hat{x})=W^{f}(\xi, \bar{x})$ where $\hat{x}$ is the only minimizer of $W^{f}(\xi, \cdot)$ in $K$. Consequently, one gets $\hat{x}=\bar{x}$. Since $\bar{x}$ is an arbitrary weak accumulation point of $\left\{\hat{x}^{n}\right\}_{n \in \mathbb{N}}$, one obtains (4.24).

In order to prove (4.23) observe that, since $w-\lim _{n \rightarrow \infty} \hat{x}^{n}=\hat{x}$, one can use (4.30) again in order to deduce that

$$
\begin{aligned}
W^{f}(\xi, \hat{x}) & \leq \liminf _{n \rightarrow \infty} W^{f}\left(\xi^{n}, \hat{x}^{n}\right) \leq \limsup _{n \rightarrow \infty} W^{f}\left(\xi^{n}, \hat{x}^{n}\right) \\
& \leq \lim _{n \rightarrow \infty} W^{f}\left(\xi^{n}, v^{n}\right)=W^{f}(\xi, \hat{x}) .
\end{aligned}
$$

This shows that $\lim _{n \rightarrow \infty} W^{f}\left(\xi^{n}, \hat{x}^{n}\right)=W^{f}(\xi, \hat{x})$, that is, (4.23) holds. 
4.7. A direct consequence of Theorem 4.5 is the monotonicity of the operator $P_{K}^{f}$.

Proposition 4.6. Suppose that $f$ is either strongly coercive or totally convex at each point of its domain. Then, for any nonempty closed convex subset $K$ of $\operatorname{dom} f$, the operator $P_{K}^{f}$ monotone. Moreover, if $\operatorname{dom} P_{K}^{f}=X^{*}$ and $K \subseteq \operatorname{int}(\operatorname{dom} f)$, then $P_{K}^{f}$ is maximal monotone.

Proof. According to the definition of $P_{K}^{f}$, for any $\xi, \zeta \in \operatorname{dom} P_{K}^{f}$, one has that

$$
\begin{aligned}
& W^{f}\left(\xi, P_{K}^{f}(\xi)\right) \leq W^{f}\left(\xi, P_{K}^{f}(\zeta)\right), \\
& W^{f}\left(\zeta, P_{K}^{f}(\zeta)\right) \leq W^{f}\left(\zeta, P_{K}^{f}(\xi)\right) .
\end{aligned}
$$

Adding these inequalities one obtains

$$
\left\langle P_{K}^{f}(\xi)-P_{K}^{f}(\zeta), \xi-\zeta\right\rangle \geq 0
$$

showing the operator $P_{K}^{f}$ is monotone. According to Theorem 4.5, the monotone operator $P_{K}^{f}$ is hemicontinuous. Now, suppose that $\operatorname{dom} P_{K}^{f}=X^{*}$ and $K \subseteq \operatorname{int}(\operatorname{dom} f)$. Applying [56, Corollary 2.3, page 106], maximal monotonicity of $P_{K}^{f}$ results.

4.8. Computing relative projections may not be an easy task. Clearly, for any $\xi \in \operatorname{int}($ dom $\left.f^{*}\right)$, we have

$$
P_{X}^{f}(\xi)=f^{* \prime}(\xi)
$$

because

$$
W^{f}\left(\xi, f^{* \prime}(\xi)\right)=f^{*}(\xi)-\left\langle\xi, f^{* \prime}(\xi)\right\rangle+f\left(f^{* \prime}(\xi)\right)
$$

It is easy to see that (4.10) applies with $f$ and $f^{*}$ interchanged, because $f=f^{* *}$. If $K$ is a hyperplane or a half space, then formulas for computing the Bregman projection $P_{K}^{f}\left(f^{\prime}(x)\right)=\Pi_{K}^{f}(x)$ are given in $[6,30]$ in the case when $f=\|\cdot\|^{p}$ for some $p \in(1, \infty)$ and $X$ is smooth and strictly convex. Iterative algorithms for computing Bregman projections on more general sets are presented in [15]. The following result gives a formula for computing relative projections onto closed hyperplanes and half spaces. It applies, for instance, when $X$ is smooth and $f=\|\cdot\|^{p}$ with $p \in(1, \infty)$.

Theorem 4.7. Suppose that $\operatorname{dom} f=X, f$ is totally convex at any $x \in X, \operatorname{dom} f^{*}=X^{*}$, $f^{* \prime}$ is positively homogeneous of degree $q>0, a \in X^{*} \backslash\{0\}$ and $b \in \mathbb{R}$.

(i) If $K=\{x \in X:\langle a, x\rangle=b\}$, then

$$
P_{K}^{f}(\xi)=f^{* \prime}(s a+\xi),
$$

where $s$ is a necessarily existing solution of the equation

$$
\left\langle a, f^{* \prime}(s a+\xi)\right\rangle=b .
$$


(ii) If $K=\{x \in X:\langle a, x\rangle \geq b\}$, then

$$
P_{K}^{f}(\xi)= \begin{cases}f^{*^{\prime}}(s a+\xi) & \text { if } f^{*^{\prime}}(\xi) \notin K \\ f^{*^{\prime}}(\xi) & \text { if } f^{*^{\prime}}(\xi) \in K,\end{cases}
$$

where $s$ is a necessarily existing positive solution of (4.38).

Proof. We first justify that $\operatorname{dom} f^{* \prime}=X^{*}$. Indeed, this is so because the function $f$ is essentially strictly convex (cf. Proposition 2.13) and, according to [13, Theorem 5.9], it satisfies $\operatorname{dom} f^{* \prime}=\operatorname{int}\left(\operatorname{dom} f^{*}\right)$ which is exactly $X^{*}$ in our context.

Now we show that there exists $s \in \mathbb{R}$ such that $\left\langle a, f^{* \prime}(s a+\xi)\right\rangle=b$. To this end, consider the function

$$
\psi(s)=\left\langle a, f^{* \prime}(s a+\xi)\right\rangle-b .
$$

Clearly, $\psi(0)=\left\langle a, f^{* \prime}(\xi)\right\rangle-b$. If $f^{*^{\prime}}(\xi) \in K$, then $s=0$ is the required solution. Otherwise, suppose that $\psi(0)<0$ and observe that the function $\psi$ is continuous. By the homogeneity of $f^{*^{\prime}}$ one has $f^{*^{\prime}}(0)=0^{q} f^{*^{\prime}}(0)=0$ and, thus, one obtains that

$$
\left\langle a, f^{* \prime}(a)\right\rangle=\left\langle a-0, f^{*^{\prime}}(a)-f^{*^{\prime}}(0)\right\rangle>0,
$$

due to the strict monotonicity of $f^{* \prime}$. Consequently, for any $s>0$, one has

$$
\psi(s)=s^{q}\left\langle a, f^{* \prime}\left(a+s^{-1} \xi\right)\right\rangle-b .
$$

Taking into account that $f^{* \prime}$ is norm-to-weak* continuous, one deduces that

$$
\lim _{s \rightarrow \infty} \psi(s)=\left\langle a, f^{* \prime}(a)\right\rangle \lim _{s \rightarrow \infty} s^{q}-b=\infty .
$$

This, the fact that $\psi(0)<0$, and the continuity of $\psi$ imply that $\psi$ has a root $s$ in the interval $(0, \infty)$. Now, observe that for the root $s$ of $\psi$ and for all $y \in K$ one has

$$
\begin{aligned}
\left\langle\xi-f^{\prime}\left(f^{* \prime}(s a+\xi)\right), f^{* \prime}(s a+\xi)-y\right\rangle & =\left\langle\xi-(s a+\xi), f^{* \prime}(s a+\xi)-y\right\rangle \\
& =-s\left\langle a, f^{* \prime}(s a+\xi)-y\right\rangle=0,
\end{aligned}
$$

showing that $f^{* \prime}(s a+\xi)$ satisfies the variational inequality (4.14). The case $\psi(0)>0$ can be treated similarly by noting that it is exactly the previous case with $a$ and $b$ replaced by $-a$ and $-b$, respectively. This proves (i). The proof of (ii) is similar.

\section{A method of solving operator equations}

5.1. In this section we assume that $X$ is a reflexive Banach space and $f: X \rightarrow \mathbb{R}$ is a lower semicontinuous function satisfying the following conditions:

Assumption 5.1. (i) $f$ is totally convex on bounded sets.

(ii) $f$, as well as its Fenchel conjugate $f^{*}$, are defined and (Gâteaux) differentiable on $X$ and $X^{*}$, respectively.

(iii) $f^{\prime}$ is uniformly continuous and $f^{*}$ is bounded on bounded sets. 
Note that, since $f$ is lower semicontinuous, then the differentiability of $f^{*}$ required by Assumption 5.1(ii) can be deduced from the strict convexity of $f$ which is guaranteed by Assumption 5.1(i) (cf. [64, Theorem 11.13]). In particular, this happens when $X$ is finite dimensional because, in this case, $f$ is continuous on $X$ as being convex.

We consider the problem of finding a vector $x$ such that

$$
A x=0, \quad x \in \Omega,
$$

where $A: \operatorname{dom} A \rightarrow X^{*}$ is a given operator and $\Omega$ is a nonempty closed convex subset of $\operatorname{dom} A$. We are going to present an iterative method for solving (5.1) for a class of operators which, when $X$ is a Hilbert space, contains all inverse-strongly-monotone operators (see [53] and Lemma 5.3 below) and, implicitly, the gradients of many differentiable functions of interest in optimization (see [11, Corollary 10]).

For each $\alpha \in(0, \infty)$ we define the operator $A_{\alpha}^{f}: \operatorname{dom} A \rightarrow X$ by

$$
A_{\alpha}^{f} x=f^{* \prime}\left(f^{\prime}(x)-\alpha A x\right) .
$$

Note that $A x=0$ if and only if $x \in \operatorname{dom} A$ is a fixed point of $A_{\alpha}^{f}$. We say that the operator $A$ is inverse-monotone relative to $f$ on the set $\Omega$ if there exists a real number $\alpha>0$ and a vector $z \in \Omega$ such that

$$
\left\langle A y, A_{\alpha}^{f} y-z\right\rangle \geq 0, \quad \forall y \in \Omega .
$$

In this case, the vector $z$ involved in (5.3) is called monotonicity pole of $A$.

5.2. The most typical example of an operator which is inverse-monotone relative to $f$ is the operator $A=f^{\prime}$ in the case when the function $f$ has a global minimizer $z \in X$. In this case, for any $\alpha \in(0,1)$, we have that

$$
A_{\alpha}^{f} x=f^{* \prime}\left((1-\alpha) f^{\prime}(x)\right)=\left((1-\alpha)^{-1} f\right)^{* \prime}\left(f^{\prime}(x)\right) .
$$

Also, according to $[64$, Theorem $11.8(\mathrm{~b})]$, we have that $\left((1-\alpha)^{-1} f\right)^{*^{\prime}}(0)=z$ because $z$ also minimizes the function $(1-\alpha)^{-1} f$ on $X$. Hence,

$$
\begin{aligned}
\left\langle A x, A_{\alpha}^{f} x-z\right\rangle & =\left\langle f^{\prime}(x),\left(\frac{1}{1-\alpha} f\right)^{*}\left(f^{\prime}(x)\right)-z\right\rangle \\
& =\left\langle f^{\prime}(x)-0,\left(\frac{1}{1-\alpha} f\right)^{*}\left(f^{\prime}(x)\right)-\left(\frac{1}{1-\alpha} f\right)^{*}(0)\right\rangle \geq 0,
\end{aligned}
$$

where the last inequality is due to the monotonicity of the operator $\left((1-\alpha)^{-1} f\right)^{* \prime}$.

5.3. The following result establishes connections between the properties of relative inverse-monotonicity defined here and the property of total nonexpansivity introduced in [28]. Recall that an operator $T: X \rightarrow X$ is called totally nonexpansive with respect to the function $f$ on the set $\Omega$ if there exists a vector $z \in \Omega$ such that

$$
D_{f}(z, T x)+D_{f}(T x, x) \leq D_{f}(z, x), \quad \forall x \in \Omega .
$$


A vector $z$ for which the condition (5.6) is satisfied is called nonexpansivity pole of $T$ with respect to $f$ and it is necessarily a fixed point of $T$. Note that total nonexpansivity of operators is closely related to the notion of $\mathscr{B}$-class operator in the sense given to this term by Bauschke et al. [14]. More precisely, if a $\mathscr{B}$-class operator $T$ has fixed points, then it is totally nonexpansive.

Lemma 5.2. The operator $A$ is inverse-monotone relative to $f$ on $\Omega$ with constant $\alpha$ if and only if the operator $A_{\alpha}^{f}$ is totally nonexpansive with respect to fon $\Omega$, that is, for some $z \in \Omega$, the following inequality holds:

$$
D_{f}\left(z, A_{\alpha}^{f} x\right)+D_{f}\left(A_{\alpha}^{f} x, x\right) \leq D_{f}(z, x), \quad \forall x \in \Omega .
$$

In this case, $z \in \Omega$ is a monotonicity pole of $A$ if and only if it is a nonexpansivity pole of $A_{\alpha}^{f}$. Proof. Observe that for any $x \in \Omega$ one has

$$
D_{f}(z, x)-D_{f}\left(z, A_{\alpha}^{f} x\right)=f\left(A_{\alpha}^{f} x\right)-f(x)-\left\langle f^{\prime}(x), z-x\right\rangle+\left\langle f^{\prime}\left(A_{\alpha}^{f} x\right), z-A_{\alpha}^{f} x\right\rangle,
$$

where $f^{\prime}\left(A_{\alpha}^{f} x\right)=f^{\prime}(x)-\alpha A x$. Hence,

$$
D_{f}(z, x)-D_{f}\left(z, A_{\alpha}^{f} x\right)=D_{f}\left(A_{\alpha}^{f} x, x\right)+\alpha\left\langle A x, A_{\alpha}^{f} x-z\right\rangle .
$$

This implies that $A$ is inverse-monotone relative to $f$ on $\Omega$ and $z$ is a monotonicity pole of it if and only if $A_{\alpha}^{f} x$ is totally nonexpansive with respect to $f$ on $\Omega$ and $z$ is a nonexpansivity pole of it.

5.4. It is interesting to observe that if $X$ is a Hilbert space and if $f(x)=(1 / 2)\|x\|^{2}$, then the inverse-monotonicity condition (5.3) can be rewritten in a more familiar form. Operators $B$ on a Hilbert space $X$ which satisfy the condition that there exists a real number $\alpha>0$ such that

$$
\langle B x-B z, x-z\rangle \geq \alpha\|B x-B z\|^{2}, \quad \forall x, z \in \operatorname{dom} B
$$

are called inverse-strongly-monotone operators (see, for instance, [53, page 320]). These are nonexpansive operators whose (set valued) inverses are strongly monotone. Note that, according to the lemma proved below, an inverse-strongly-monotone operator $B$ which has a zero at some point $z \in \operatorname{dom} B$ is necessarily inverse-monotone relative to $f=(1 / 2)\|\cdot\|^{2}$ on $\operatorname{dom} B$ and has $z$ as a monotonicity pole. In other words, the notion of inverse-monotonicity relative to a function $f$ can be viewed as a generalization of that of inverse-strong-monotonicity for operators in Hilbert spaces.

Lemma 5.3. If $X$ is a Hilbert space and $f=(1 / 2)\|\cdot\|^{2}$, then the operator $A$ is inversemonotone relative to $f$ on $\Omega$ if and only if, for some $z \in \Omega$, one has that $A z=0$ and

$$
\langle A x, x-z\rangle \geq \alpha\|A x\|^{2}, \quad \forall x \in \Omega .
$$


Proof. If $A$ is inverse-monotone relative to $f$ on $\Omega$ and $z \in \Omega$ is a monotonicity pole of it then, by Lemma 5.2, $z$ is a nonexpansivity pole of $A_{\alpha}^{f}$ and, thus, $z$ is a fixed point of $A_{\alpha}^{f}$. Since, in these circumstances, $f^{\prime}$ is the identity, it follows that $z=A_{\alpha}^{f} z=z-\alpha A z$ and, therefore, $z$ is a solution of (5.1). Hence, for any $x \in \Omega$, one has

$$
\begin{aligned}
\langle A x, x-z\rangle-\alpha\|A x\|^{2} & =\langle A x-A z, x-z\rangle-\alpha\|A x-A z\|^{2} \\
& =\langle A x-A z, x-z-\alpha(A x-A z)\rangle \\
& =\left\langle A x, A_{\alpha}^{f} x-z\right\rangle \geq 0 .
\end{aligned}
$$

Conversely, if $A$ satisfies (5.11) for some $z \in \Omega$ with $A z=0$ and for any $x \in \Omega$, then the previous chain of equalities shows that (5.3) holds.

5.5. Now we are going to describe an iterative procedure for solving (5.1). To this end, for each real number $\alpha>0$, we define the operator $S_{\alpha}^{f}: \operatorname{dom} A \rightarrow X$ by

$$
S_{\alpha}^{f} x=P_{\Omega}^{f}\left(f^{\prime}(x)-\alpha A x\right),
$$

where $P_{\Omega}^{f}$ is the projection operator relative to $f$ onto the set $\Omega$. According to (4.13) we also have

$$
S_{\alpha}^{f}=\Pi_{\Omega}^{f} \circ A_{\alpha}^{f} .
$$

We are interested in the behavior of the following iterative process:

$$
\text { Choose } y^{0} \in \operatorname{dom} A \text { and define } y^{k+1}=S_{\alpha}^{f} y^{k}, \quad \forall k \in \mathbb{N} \text {. }
$$

The next result shows that if the operator $A$ is inverse-monotone relative to a function $f$ having the properties required at the beginning of this section, then (5.1) has solutions and the sequences $\left\{y^{k}\right\}_{k \in \mathbb{N}}$ generated according to the rule (5.15) produces weak approximations of solutions of (5.1). A careful analysis of the proof shows that (5.15) is essentially a method of approximating fixed points of the operator $S_{\alpha}^{f}$.

THEOREM 5.4. If the operator $A$ is inverse-monotone relative to $f$ on $\Omega$ with constant $\alpha>0$, then (5.1) has solutions and any sequence $\left\{y^{k}\right\}_{k \in \mathbb{N}}$ generated according to the rule (5.15) is bounded, has weak accumulation points and $\lim _{k \rightarrow \infty} A y^{k}=0$. If, in addition, the operator $A$ is sequentially weakly-weakly* continuous on $\Omega$, then any weak accumulation point of $\left\{y^{k}\right\}_{k \in \mathbb{N}}$ is a solution of (5.1). In this case, if (5.1) has unique solution, then the sequence $\left\{y^{k}\right\}_{k \in \mathbb{N}}$ converges weakly to that solution.

Proof. According to Lemma 5.2, the operator $A_{\alpha}^{f}$ is totally nonexpansive with respect to $f$ on $\Omega$. This implies that there exists a vector $z \in \Omega$ such that

$$
D_{f}\left(z, A_{\alpha}^{f} x\right) \leq D_{f}(z, x), \quad \forall x \in \Omega
$$

Taking here $x=z$, one obtains that $z=A_{\alpha}^{f} z$. Consequently, one has $f^{\prime}(z)=f^{\prime}\left(A_{\alpha}^{f} z\right)$ which, together with (5.2), implies $A z=0$. Hence, (5.1) has solutions. According to (5.16) 
combined with Corollary 4.4 and (5.14) one has that

$$
D_{f}\left(z, S_{\alpha}^{f} x\right)+D_{f}\left(S_{\alpha}^{f} x, A_{\alpha}^{f} x\right) \leq D_{f}\left(z, A_{\alpha}^{f} x\right) \leq D_{f}(z, x), \quad \forall x \in \Omega .
$$

Writing these inequalities for $x=y^{k}$ (which is contained in $\Omega$ ) one deduces that

$$
D_{f}\left(z, y^{k+1}\right)+D_{f}\left(y^{k+1}, A_{\alpha}^{f} y^{k}\right) \leq D_{f}\left(z, A_{\alpha}^{f} y^{k}\right) \leq D_{f}\left(z, y^{k}\right), \quad \forall k \in \mathbb{N} .
$$

This implies that the sequence $\left\{D_{f}\left(z, y^{k}\right)\right\}_{k \in \mathbb{N}}$ is nonincreasing and, therefore, bounded. Thus, the sequence $\left\{f^{\prime}\left(y^{k}\right)\right\}_{\{k \in \mathbb{N}\}}$ is contained in the set $R_{\beta}^{f^{*}}(z)$, where $\beta$ is a positive upper bound of the sequence $\left\{D_{f}\left(z, y^{k}\right)\right\}_{k \in \mathbb{N}}$. The set $R_{\beta}^{f^{*}}(z)$ is bounded because $f^{*}-\langle\cdot, z\rangle$ is coercive and, thus, $y^{k}=f^{* \prime}\left(f^{\prime}\left(y^{k}\right)\right)$ is bounded (cf. Assumption 5.1). Hence, the sequence $\left\{y^{k}\right\}_{k \in \mathbb{N}}$ is bounded and, since the space $X$ is reflexive, there exists a subsequence $\left\{y^{i_{k}}\right\}_{k \in \mathbb{N}}$ of $\left\{y^{k}\right\}_{k \in \mathbb{N}}$ which converges weakly to some point $\bar{y}$. Since all $y^{i_{k}}$ 's are contained in $\Omega$, the vector $\bar{y}$ is contained in $\Omega$ too ( $\Omega$ is convex and closed and, thus, weakly closed). We claim that the sequences $\left\{y^{i_{k}}\right\}_{k \in \mathbb{N}}$ and $\left\{A_{\alpha}^{f} y^{i_{k}}\right\}_{k \in \mathbb{N}}$ have the same weak limit $\bar{y}$. For proving this it is sufficient to show that

$$
\lim _{k \rightarrow \infty}\left\|A_{\alpha}^{f} y^{k}-y^{k}\right\|=0 .
$$

Observe that (5.18) implies

$$
0 \leq D_{f}\left(z, A_{\alpha}^{f} y^{k}\right)-D_{f}\left(z, y^{k+1}\right) \leq D_{f}\left(z, y^{k}\right)-D_{f}\left(z, y^{k+1}\right), \quad \forall k \in \mathbb{N} .
$$

As noted above, the sequence $\left\{D_{f}\left(z, y^{k}\right)\right\}_{k \in \mathbb{N}}$ converges. Therefore, the last inequality shows that the sequence $\left\{D_{f}\left(z, A_{\alpha}^{f} y^{k}\right)\right\}_{k \in \mathbb{N}}$ converges and has the same limit as the sequence $\left\{D_{f}\left(z, y^{k}\right)\right\}_{k \in \mathbb{N}}$. By (5.9) one also has

$$
D_{f}\left(z, y^{k}\right)-D_{f}\left(z, A_{\alpha}^{f} y^{k}\right)=D_{f}\left(A_{\alpha}^{f} y^{k}, y^{k}\right)+\alpha\left\langle A y^{k}, A_{\alpha}^{f} y^{k}-z\right\rangle,
$$

where the last term on the right-hand side is nonnegative because of the inverse- monotonicity of $A$. Thus, one obtains that

$$
0 \leq \lim _{k \rightarrow \infty} D_{f}\left(A_{\alpha}^{f} y^{k}, y^{k}\right) \leq \lim _{k \rightarrow \infty}\left[D_{f}\left(z, y^{k}\right)-D_{f}\left(z, A_{\alpha}^{f} y^{k}\right)\right]=0
$$

which shows that $\lim _{k \rightarrow \infty} D_{f}\left(A_{\alpha}^{f} y^{k}, y^{k}\right)=0$. The function $f$ is totally convex on bounded sets and the sequence $\left\{y^{k}\right\}_{k \in \mathbb{N}}$ is bounded. Therefore, the function $v_{f}(E, \cdot)$ associated to the set $E:=\left\{y^{k}\right\}_{k \in \mathbb{N}}$, is strictly increasing on $[0, \infty)$ (see Section 2.10), vanishes at 0 and satisfies

$$
\nu_{f}\left(E,\left\|A_{\alpha}^{f} y^{k}-y^{k}\right\|\right) \leq D_{f}\left(A_{\alpha}^{f} y^{k}, y^{k}\right), \quad \forall k \in \mathbb{N} .
$$

This and (5.22) imply that

$$
\lim _{k \rightarrow \infty} v_{f}\left(E,\left\|A_{\alpha}^{f} y^{k}-y^{k}\right\|\right)=0
$$


Due to the strict monotonicity of $v_{f}(E, \cdot)$, this cannot happen unless (5.19) holds. Since $f^{\prime}$ is uniformly continuous on bounded sets, by (5.19) one deduces that

$$
\lim _{k \rightarrow \infty}\left\|f^{\prime}\left(A_{\alpha}^{f} y^{k}\right)-f^{\prime}\left(y^{k}\right)\right\|_{*}=0
$$

Note that, according to (5.2), one also has that $f^{\prime}\left(A_{\alpha}^{f} y^{k}\right)-f^{\prime}\left(y^{k}\right)=-\alpha A y^{k}$. The last two equalities imply that $\lim _{k \rightarrow \infty}\left\|A y^{k}\right\|_{*}=0$.

Now, suppose that $A$ is sequentially weakly-weakly* continuous. Since the sequence $\left\{y^{i_{k}}\right\}_{k \in \mathbb{N}}$ converges weakly to $\bar{y}$, we obtain that $\|A \bar{y}\|_{*} \leq \lim _{k \rightarrow \infty}\left\|A y^{i_{k}}\right\|_{*}=0$, showing that $\bar{y}$ is a solution of (5.1). If the solution of (5.1) is unique, then the sequence $\left\{y^{k}\right\}_{k \in \mathbb{N}}$ has a unique weak accumulation point and this completes the proof.

5.6. The sequential weak-weak* continuity condition imposed upon the operator $A$ in Theorem 5.4 is restrictive. However, this condition always holds when $A$ is linear and continuous on $X$ or, alternatively, when $X$ is finite dimensional and $A$ is continuous on its domain. An immediate application of Theorem 5.4 to convex unconstrained optimization in $\mathbb{R}^{n}$ is emphasized in the result below.

Corollary 5.5. Suppose that $f: \mathbb{R}^{n} \rightarrow \mathbb{R}$ is a differentiable strictly convex function. If $f^{\prime}$ is uniformly continuous on bounded sets and if $f$ has a global minimizer, then for each $\alpha \in(0,1)$, the sequence $\left\{y^{k}\right\}_{k \in \mathbb{N}}$ generated according to the rule

$$
y^{0} \in \mathbb{R}^{n}, \quad y^{k+1}=f^{* \prime}\left((1-\alpha) f^{\prime}\left(y^{k}\right)\right), \quad \forall k \in \mathbb{N},
$$

converges to the minimizer of $f$.

Proof. Theorem 2.14 ensures that $f$ satisfies Assumption 5.1. As noted in Section 5.2, in these circumstances, the operator $A=f^{\prime}$ is inverse-monotone relative to $f$. Applying Theorem 5.4 and taking into account that $f$ has a single minimizer (i.e., the equation $f^{\prime}(x)=0$ has a single solution) yields the conclusion.

5.7. The following technical result, which will be used later, is a generalization of a result known as Opial's lemma [55, Lemma 2]. Opial's lemma says that if $X$ is a Hilbert space, if $g=(1 / 2)\|\cdot\|^{2}$ and if $T: \Omega \rightarrow X$ is a nonexpansive mapping on the nonempty closed convex subset $\Omega$ of $X$, then for any sequence $\left\{z^{k}\right\}_{k \in \mathbb{N}} \subseteq \Omega$ which is weakly convergent and has $\lim _{k \rightarrow \infty}\left\|T z^{k}-z^{k}\right\|=0$, the vector $z=w-\lim _{k \rightarrow \infty} z^{k}$ is necessarily a fixed point of $T$. We extend this result to not necessarily Hilbertian Banach spaces and to nonexpansive operators in a generalized sense.

Lemma 5.6. Suppose that the function $g: X \rightarrow \mathbb{R}$ is totally convex on bounded sets and has bounded on bounded sets Gâteaux derivative $g^{\prime}$. If $T: \Omega \rightarrow X$ is an operator such that

$$
D_{g}(T y, T x) \leq D_{g}(y, x), \quad \forall x, y \in \Omega,
$$

then for any weakly convergent sequence $\left\{z^{k}\right\}_{k \in \mathbb{N}} \subseteq \Omega$ which has $\lim _{k \rightarrow \infty} D_{g}\left(T z^{k}, z^{k}\right)=0$, the vector $z=w-\lim _{k \rightarrow \infty} z^{k}$ is a fixed point of $T$. 
Proof. Observe that, for any $x \in X$, one has

$$
\begin{aligned}
D_{g}\left(z^{k}, x\right)-D_{g}\left(z^{k}, z\right) & =-g(x)-\left\langle g^{\prime}(x), z^{k}-x\right\rangle+g(z)+\left\langle g^{\prime}(z), z^{k}-z\right\rangle \\
& =D_{g}(z, x)+\left\langle g^{\prime}(x)-g^{\prime}(z), z-z^{k}\right\rangle,
\end{aligned}
$$

where $\lim _{k \rightarrow \infty}\left\langle g^{\prime}(x)-g^{\prime}(z), z-z^{k}\right\rangle=0$. Therefore,

$$
\liminf _{k \rightarrow \infty} D_{g}\left(z^{k}, x\right) \geq D_{g}(z, x)+\liminf _{k \rightarrow \infty} D_{g}\left(z^{k}, z\right),
$$

which shows that, when $x \neq z$, one has

$$
\liminf _{k \rightarrow \infty} D_{g}\left(z^{k}, x\right)>\liminf _{k \rightarrow \infty} D_{g}\left(z^{k}, z\right)
$$

We claim that

$$
\liminf _{k \rightarrow \infty} D_{g}\left(T z^{k}, T z\right)=\liminf _{k \rightarrow \infty} D_{g}\left(z^{k}, T z\right) .
$$

In order to show that, observe that

$$
\begin{aligned}
D_{g}\left(T z^{k}, T z\right) & =g\left(T z^{k}\right)-g(T z)-\left\langle g^{\prime}(T z), T z^{k}-T z\right\rangle \\
& =D_{g}\left(z^{k}, T z\right)+\left[g\left(T z^{k}\right)-g\left(z^{k}\right)\right]+\left\langle g^{\prime}(T z), z^{k}-T z^{k}\right\rangle .
\end{aligned}
$$

The function $g$ is sequentially consistent (cf. Theorem 2.10). Consequently, since

$$
\lim _{k \rightarrow \infty} D_{g}\left(T z^{k}, z^{k}\right)=0
$$

one also has that

$$
\lim _{k \rightarrow \infty}\left\|T z^{k}-z^{k}\right\|=0
$$

This implies that

$$
\lim _{k \rightarrow \infty}\left\langle g^{\prime}(T z), z^{k}-T z^{k}\right\rangle=0
$$

From the convexity of $g$ one deduces that

$$
\left\langle g^{\prime}\left(T z^{k}\right), T z^{k}-z^{k}\right\rangle \geq g\left(T z^{k}\right)-g\left(z^{k}\right) \geq\left\langle g^{\prime}\left(z^{k}\right), T z^{k}-z^{k}\right\rangle
$$

where the sequence $\left\{z^{k}\right\}_{k \in \mathbb{N}}$ is bounded as being weakly convergent and $\left\{T z^{k}\right\}_{k \in \mathbb{N}}$ is bounded because of (5.34). The function $g^{\prime}$ being bounded on bounded sets the sequences $\left\{g^{\prime}\left(z^{k}\right)\right\}_{k \in \mathbb{N}}$ and $\left\{g^{\prime}\left(T z^{k}\right)\right\}_{k \in \mathbb{N}}$ are bounded too. Thus, letting $k \rightarrow \infty$ in (5.36) we deduce that

$$
\lim _{k \rightarrow \infty}\left[g\left(T z^{k}\right)-g\left(z^{k}\right)\right]=0 .
$$


This, combined with (5.35) and (5.32), proves (5.31). Now, suppose by contradiction that $T z \neq z$. Then, according to (5.30), one has that

$$
\liminf _{k \rightarrow \infty} D_{g}\left(z^{k}, T z\right)>\liminf _{k \rightarrow \infty} D_{g}\left(z^{k}, z\right) .
$$

By (5.27), one deduces that

$$
\liminf _{k \rightarrow \infty} D_{g}\left(z^{k}, z\right) \geq \liminf _{k \rightarrow \infty} D_{g}\left(T z^{k}, T z\right)=\liminf _{k \rightarrow \infty} D_{g}\left(z^{k}, T z\right),
$$

where the last equality results from (5.31). Since (5.38) and (5.39) contradict each other, our assumption that $T z \neq z$ is false, that is, $z$ is a fixed point of $T$.

5.8. It was noted above that applicability of Theorem 5.4 is restricted because of the requirement that the operator $A$ should be sequentially weakly-weakly* continuous. Using the generalization of Opial's lemma proved above we can give another variant of Theorem 5.4 in which that requirement is dropped. The price to pay for dropping that condition is the need to strengthen our demands on the function $f$ and on the nonexpansivity of $A$. In order to present the alternative version of Theorem 5.4 we will call the operator $A$ inverse-strongly-monotone on $\Omega$ relative to $f$ with constant $\alpha>0$ if $A$ is inversemonotone relative to $f$ with constant $\alpha>0$ and the operator $A_{\alpha}^{f}$ is nonexpansive on $\Omega$ with respect to $f$ in the sense given to this term in [32], that is, if

$$
D_{f}\left(A_{\alpha}^{f} x, A_{\alpha}^{f} y\right) \leq D_{f}(x, y), \quad \forall x, y \in \Omega .
$$

Note that, in general, the operator $A$ may satisfy condition (5.40) for some $\alpha>0$ without being inverse-monotone with respect to $f$ with constant $\alpha$. For instance, take $X$ to be a Hilbert space, $\Omega=X, f=(1 / 2)\|\cdot\|^{2}$ and $A x=x$ for any $x \in X$. It is easy to verify that, in this case, condition (5.40) holds for any $\alpha \in(0,2]$. However, the operator $A$ is not inverse-monotone relative to $f$ (i.e., it does not satisfy (5.3)) for any $\alpha \in(1,2]$.

Now we are in position to prove the new version of Theorem 5.4 that applies to operators which are inverse-strongly-monotone with respect to $f$.

THEOREM 5.7. If the operator $A$ is inverse-strongly-monotone relative to $f$ on $\Omega$ with constant $\alpha>0$ and if (5.1) has at least one solution, then any sequence $\left\{y^{k}\right\}_{k \in \mathbb{N}}$ generated according to the rule (5.15) has the following properties:

(i) it is bounded, has weak accumulation points and any such point is a solution of (5.1);

(ii) if (5.1) has unique solution, then the sequence $\left\{y^{k}\right\}_{k \in \mathbb{N}}$ converges weakly to that solution;

(iii) if the space $X$ has finite dimension, then $\left\{y^{k}\right\}_{k \in \mathbb{N}}$ converges to a solution of (5.1).

Proof. Let $z \in \Omega$ be a solution of (5.1). Then $z$ is a fixed point of $A_{\alpha}^{f}$ and one has

$$
D_{f}\left(z, A_{\alpha}^{f} x\right)=D_{f}\left(A_{\alpha}^{f} z, A_{\alpha}^{f} x\right) \leq D_{f}(z, x), \quad \forall x \in \Omega .
$$

Consequently, (5.16) holds and all the considerations in the proof of Theorem 5.4 up to, and including, formula (5.22) can be reproduced without any modification. Formula 
(5.22) implies that

$$
\lim _{k \rightarrow \infty} D_{f}\left(A_{\alpha}^{f} y^{k}, y^{k}\right)=0
$$

Also, according to [48, Proposition 4], the derivative $f^{\prime}$ is bounded on bounded sets because it is uniformly continuous on bounded sets (cf. Assumption 5.1). Therefore, one can apply Lemma 5.6 to the weakly convergent subsequence $\left\{y^{i_{k}}\right\}_{k \in \mathbb{N}}$. It implies that $\bar{y}$ is a fixed point of the operator $A_{\alpha}^{f}$, that is, a solution of the problem (5.1). This proves (i). Clearly, (ii) results from (i). Now, suppose that $X$ has finite dimension. By Corollary 4.4 and (5.41), one has that

$$
D_{f}\left(\bar{y}, y^{k+1}\right)+D_{f}\left(y^{k+1}, A_{\alpha}^{f} y^{k}\right) \leq D_{f}\left(\bar{y}, y^{k}\right), \quad \forall k \in \mathbb{N} .
$$

This implies that $\left\{D_{f}\left(\bar{y}, y^{k}\right)\right\}_{k \in \mathbb{N}}$ is convergent. Since it has a subsequence convergent to zero, then the whole sequence converges to zero. By Assumption 5.1 and Theorem 2.10, $f$ is sequentially consistent. Hence, $\left\|y^{k}-\bar{y}\right\| \rightarrow 0$ as $k \rightarrow \infty$ and this completes the proof.

5.9. Theorem 5.7 leads to the following partially known result (see [1], [17, Section 2.3] and [34, Corollary 4.1]).

Corollary 5.8. Suppose that $X$ is a Hilbert space and $\varphi: X \rightarrow \mathbb{R}$ is a convex function which is Gâteaux differentiable on $X$ and such that its derivative is Lipschitzian with Lipschitz constant $L$. If $\varphi$ has at least one minimizer in $X$, then any sequence generated according to the rule

$$
y^{k+1}=y^{k}-\alpha \varphi^{\prime}\left(y^{k}\right), \quad \forall k \in \mathbb{N},
$$

has the following properties, no matter how the constant $\alpha \in\left(0, L^{-1}\right]$ and the initial point $y^{0} \in X$ are chosen:

(i) $\left\{y^{k}\right\}_{k \in \mathbb{N}}$ is bounded, has weak accumulation points, and any such point is a minimizer of $\varphi$;

(ii) if $\varphi$ has a unique minimum on $X$, then the sequence $\left\{y^{k}\right\}_{k \in \mathbb{N}}$ converges weakly to a minimizer of $\varphi$;

(iii) if $X$ is finite dimensional, then the sequence $\left\{y^{k}\right\}_{k \in \mathbb{N}}$ converges to a minimizer of $\varphi$.

Proof. According to [11, Corollary 10], the operator $A=\varphi^{\prime}$ is inverse-strongly-monotone with any constant $\alpha \in\left(0, L^{-1}\right]$. By Lemma 5.3 , this implies that $\varphi^{\prime}$ is inverse-monotone relative to the function $f=(1 / 2)\|\cdot\|^{2}$ on the whole space $X$ with any constant $\alpha \in$ $\left(0, L^{-1}\right]$. Also, one can easily check that $A$ satisfies condition (5.40) for any $\alpha \in\left(0,2 L^{-1}\right]$. Therefore, the operator $A$ is inverse-strongly-monotone relative to $f=(1 / 2)\|\cdot\|^{2}$ with any $\alpha \in\left(0, L^{-1}\right]$. By applying Theorem 5.7 to $A=\varphi^{\prime}$ and by taking into account that $f^{\prime}$ is the identity, the result follows. 


\section{Acknowledgments}

The authors wish to thank Anca Croitoru and Constantin Zălinescu for valuable suggestions concerning some topics discussed in this work. Also, the authors are grateful to Yakov Alber, Gábor Kassay Simeon Reich, and a referee for comments leading to substantial improvements of an earlier version of the paper. Elena Resmerita's research contribution to this work was done in part during her visit (September-December 2003) within the Institute for Pure and Applied Mathematics, UCLA, California.

\section{References}

[1] Y. I. Alber, Some iterative methods for nonregular minimization problems and estimates of the speed of convergence, Technicheskaya Kybernetika 3 (1988), 22-30 (Russian).

[2] __ Generalized projection operators in Banach spaces: properties and applications, Functional-Differential Equations (M. E. Drakhlin and E. Litsyn, eds.), Funct. Differential Equations Israel Sem., vol. 1, Coll. Judea Samaria, Ariel, 1993, pp. 1-21.

[3] _. Metric and generalized projection operators in Banach spaces: properties and applications, Theory and Applications of Nonlinear Operators of Accretive and Monotone Type, Lecture Notes in Pure and Appl. Math., vol. 178, Dekker, New York, 1996, pp. 15-50.

[4] _ Generalized projections, decompositions, and the Pythagorean-type theorem in Banach spaces, Applied Mathematics Letters 11 (1998), no. 6, 115-121.

[5] , Some new characteristics of Banach spaces, preprint, 2004.

[6] Y. I. Alber and D. Butnariu, Convergence of Bregman projection methods for solving consistent convex feasibility problems in reflexive Banach spaces, Journal of Optimization Theory and Applications 92 (1997), no. 1, 33-61.

[7] Y. I. Alber, D. Butnariu, and I. Ryazantseva, Regularization and resolution of monotone variational inequalities with operators given by hypomonotone approximations, Journal of Nonlinear and Convex Analysis 6 (2005), no. 1, 23-53.

[8] Y. I. Alber, A. N. Iusem, and M. V. Solodov, Minimization of nonsmooth convex functionals in Banach spaces, Journal of Convex Analysis 4 (1997), no. 2, 235-255.

[9] Y. I. Alber and S. Reich, An iterative method for solving a class of nonlinear operator equations in Banach spaces, Panamerican Mathematical Journal 4 (1994), no. 2, 39-54.

[10] E. Asplund, Averaged norms, Israel Journal of Mathematics 5 (1967), 227-233.

[11] J.-B. Baillon and G. Haddad, Quelques propriétés des opérateurs angle-bornés et n-cycliquement monotones, Israel Journal of Mathematics 26 (1977), no. 2, 137-150 (French).

[12] H. H. Bauschke and J. M. Borwein, Legendre functions and the method of random Bregman projections, Journal of Convex Analysis 4 (1997), no. 1, 27-67.

[13] H. H. Bauschke, J. M. Borwein, and P. L. Combettes, Essential smoothness, essential strict convexity, and Legendre functions in Banach spaces, Communications in Contemporary Mathematics 3 (2001), no. 4, 615-647.

[14] B Bregman monotone optimization algorithms, SIAM Journal on Control and Optimization 42 (2003), no. 2, 596-636.

[15] H. H. Bauschke and P. L. Combettes, Construction of best Bregman approximations in reflexive Banach spaces, Proceedings of the American Mathematical Society 131 (2003), no. 12, 37573766.

[16] H. H. Bauschke and A. S. Lewis, Dykstra's algorithm with Bregman projections: a convergence proof, Optimization 48 (2000), no. 4, 409-427.

[17] D. Bertsekas, Nonlinear Programming, 2nd ed., Athena Scientific, Massachusetts, 1999.

[18] J. M. Borwein and A. S. Lewis, Convergence of best entropy estimates, SIAM Journal on Optimization 1 (1991), no. 2, 191-205. 
[19] J. M. Borwein and M. A. Limber, On entropy maximization via convex programming, preprint, 1996, available at http://citeseer.ist.psu.edu/71881.html.

[20] L. M. Bregman, The relaxation method for finding common points of convex sets and its application to the solution of problems in convex programming, USSR Computational Mathematics and Mathematical Physics 7 (1967), 200-217.

[21] R. E. Bruck Jr., A strongly convergent iterative solution of $0 \in U(x)$ for a maximal monotone operator $U$ in Hilbert space, Journal of Mathematical Analysis and Applications 48 (1974), 114-126.

[22] _ An iterative solution of a variational inequality for certain monotone operators in Hilbert space, American Mathematical Society Bulletin 81 (1975), no. 5, 890-892.

[23] R. S. Burachik, Generalized proximal point methods for the variational inequality problem, Ph.D. thesis, Instituto de Mathematica Pura e Aplicada (IMPA), Rio de Janeiro, 1995.

[24] R. S. Burachik and S. Scheimberg, A proximal point method for the variational inequality problem in Banach spaces, SIAM Journal on Control and Optimization 39 (2000), no. 5, 1633-1649.

[25] D. Butnariu, Y. Censor, and S. Reich, Iterative averaging of entropic projections for solving stochastic convex feasibility problems, Computational Optimization and Applications 8 (1997), no. 1, $21-39$.

[26] D. Butnariu and A. N. Iusem, Local moduli of convexity and their application to finding almost common fixed points of measurable families of operators, Recent Developments in Optimization Theory and Nonlinear Analysis (Jerusalem, 1995) (Y. Censor and S. Reich, eds.), Contemp. Math., vol. 204, American Mathematical Society, Rhode Island, 1997, pp. 61-91.

[27] _ On a proximal point method for convex optimization in Banach spaces, Numerical Functional Analysis and Optimization 18 (1997), no. 7-8, 723-744.

[28] - Totally Convex Functions for Fixed Points Computation and Infinite Dimensional Optimization, Applied Optimization, vol. 40, Kluwer Academic, Dordrecht, 2000.

[29] D. Butnariu, A. N. Iusem, and R. S. Burachik, Iterative methods of solving stochastic convex feasibility problems and applications, Computational Optimization and Applications 15 (2000), no. 3 , 269-307.

[30] D. Butnariu, A. N. Iusem, and E. Resmerita, Total convexity for powers of the norm in uniformly convex Banach spaces, Journal of Convex Analysis 7 (2000), no. 2, 319-334.

[31] D. Butnariu, A. N. Iusem, and C. Zălinescu, On uniform convexity, total convexity and convergence of the proximal point and outer Bregman projection algorithms in Banach spaces, Journal of Convex Analysis 10 (2003), no. 1, 35-61.

[32] D. Butnariu, S. Reich, and A. J. Zaslavski, Weak convergence of orbits of nonlinear operators in reflexive Banach spaces, Numerical Functional Analysis and Optimization 24 (2003), no. 5-6, 489-508.

[33] D. Butnariu and E. Resmerita, The outer Bregman projection method for stochastic feasibility problems in Banach spaces, Inherently Parallel Algorithms in Feasibility and Optimization and Their Applications (Haifa, 2000) (D. Butnariu, Y. Censor, and S. Reich, eds.), Stud. Comput. Math., vol. 8, North-Holland, Amsterdam, 2001, pp. 69-86.

[34] C. Byrne, A unified treatment of some iterative algorithms in signal processing and image reconstruction, Inverse Problems 20 (2004), no. 1, 103-120.

[35] Y. Censor and A. Lent, An iterative row-action method for interval convex programming, Journal of Optimization Theory and Applications 34 (1981), no. 3, 321-353.

[36] Y. Censor and S. A. Zenios, Parallel Optimization. Theory, Algorithms, and Applications, Numerical Mathematics and Scientific Computation, Oxford University Press, New York, 1997.

[37] I. Cioranescu, Geometry of Banach Spaces, Duality Mappings and Nonlinear Problems, Mathematics and Its Applications, vol. 62, Kluwer Academic, Dordrecht, 1990.

[38] J. A. Clarkson, Uniformly convex spaces, Transactions of the American Mathematical Society 40 (1936), no. 3, 396-414. 
[39] A. L. Dontchev and T. Zolezzi, Well-Posed Optimization Problems, Lecture Notes in Mathematics, vol. 1543, Springer, Berlin, 1993.

[40] J. Eckstein, Nonlinear proximal point algorithms using Bregman functions, with applications to convex programming, Mathematics of Operations Research 18 (1993), no. 1, 202-226.

[41] P. P. B. Eggermont, Maximum entropy regularization for Fredholm integral equations of the first kind, SIAM Journal on Mathematical Analysis 24 (1993), no. 6, 1557-1576.

[42] I. Ekeland and R. Temam, Convex Analysis and Variational Problems, North-Holland, Amsterdam, 1976.

[43] H. W. Engl and G. Landl, Convergence rates for maximum entropy regularization, SIAM Journal on Numerical Analysis 30 (1993), no. 5, 1509-1536.

[44] K. Fan and I. Glicksberg, Some geometric properties of the spheres in a normed linear space, Duke Mathematical Journal 25 (1958), 553-568.

[45] R. Glowinski, J.-L. Lions, and R. Trémolières, Numerical Analysis of Variational Inequalities, Studies in Mathematics and Its Applications, vol. 8, North-Holland, Amsterdam, 1981.

[46] R. B. Holmes, A Course on Optimization and Best Approximation, Lecture Notes in Mathematics, Vol. 257, Springer, Berlin, 1972.

[47] T. Ibaraki, Y. Kimura, and W. Takahashi, Convergence theorems for generalized projections and maximal monotone operators in Banach spaces, Abstract and Applied Analysis 10 (2003), 621629.

[48] A. N. Iusem and R. Gárciga Otero, Inexact versions of proximal point and augmented Lagrangian algorithms in Banach spaces, Numerical Functional Analysis and Optimization 22 (2001), no. 56, 609-640.

[49] _ _ Augmented Lagrangian methods for cone-constrained convex optimization in Banach spaces, Journal of Nonlinear and Convex Analysis 3 (2002), no. 2, 155-176.

[50] M. Ǐ. Kadec', Spaces isomorphic to a locally uniformly convex space, Izvestija Vysš Učebnyh ZavedeniǔMatematika 6 (1959), no. 13, 51-57 (Russian).

[51] K. C. Kiwiel, Proximal minimization methods with generalized Bregman functions, SIAM Journal on Control and Optimization 35 (1997), no. 4, 1142-1168.

[52] L. Landweber, An iteration formula for Fredholm integral equations of the first kind, American Journal of Mathematics 73 (1951), 615-624.

[53] F. Liu and M. Z. Nashed, Regularization of nonlinear ill-posed variational inequalities and convergence rates, Set-Valued Analysis 6 (1998), no. 4, 313-344.

[54] U. Mosco, Convergence of convex sets and of solutions of variational inequalities, Advances in Mathematics 3 (1969), 510-585.

[55] Z. Opial, Weak convergence of the sequence of successive approximations for nonexpansive mappings, American Mathematical Society Bulletin 73 (1967), 591-597.

[56] D. Pascali and S. Sburlan, Nonlinear Mappings of Monotone Type, Martinus Nijhoff, The Hague; Sijthoff \& Noordhoff International, Alphen aan den Rijn, 1978.

[57] R. R. Phelps, Convex Functions, Monotone Operators and Differentiability, 2nd ed., Lecture Notes in Mathematics, vol. 1364, Springer, Berlin, 1993.

[58] B. Polyak, A general method for solving extremum problems, Doklady Akedamii Nauk SSSR 174 (1967), 593-597.

[59] S. Reich, Extension problems for accretive sets in Banach spaces, Journal of Functional Analysis 26 (1977), no. 4, 378-395.

[60] _ A weak convergence theorem for the alternating method with Bregman distances, Theory and Applications of Nonlinear Operators of Accretive and Monotone Type, Lecture Notes in Pure and Appl. Math., vol. 178, Dekker, New York, 1996, pp. 313-318.

[61] E. Resmerita, Fixed point and optimization methods in infinite dimensional Banach spaces, Ph.D. thesis, University of Haifa, Israel, 2003. 
[62] On total convexity, Bregman projections and stability in Banach spaces, Journal of Convex Analysis 11 (2004), 1-16.

[63] R. T. Rockafellar, Convex Analysis, Princeton Mathematical Series, no. 28, Princeton University Press, New Jersey, 1970.

[64] R. T. Rockafellar and R. J.-B. Wets, Variational Analysis, Grundlehren der Mathematischen Wissenschaften, vol. 317, Springer, Berlin, 1998.

[65] M. V. Solodov and B. F. Svaiter, An inexact hybrid generalized proximal point algorithm and some new results on the theory of Bregman functions, Mathematics of Operations Research 25 (2000), no. 2, 214-230.

[66] S. L. Troyanski, On locally uniformly convex and differentiable norms in certain non-separable Banach spaces, Studia Mathematica 37 (1970), 173-180.

[67] A. A. Vladimirov, Ju. E. Nesterov, and Ju. N. Čekanov, Uniformly convex functionals, Vestnik Moskovskogo Universiteta. Seriya XV. Vychislitel'naya Matematika i Kibernetika 3 (1978), 1223 (Russian).

[68] C. Zălinescu, On uniformly convex functions, Journal of Mathematical Analysis and Applications 95 (1983), no. 2, 344-374.

[69]__ Convex Analysis in General Vector Spaces, World Scientific, New Jersey, 2002.

Dan Butnariu: Department of Mathematics, University of Haifa, 31905 Haifa, Israel E-mail address: dbutnaru@math.haifa.ac.il

Elena Resmerita: Johann Radon Institute for Computational and Applied Mathematics, Austrian Academy of Sciences, Altenbergerstrasse 69, Linz 4040, Austria

E-mail address: elena.resmerita@ricam.oeaw.ac.at 


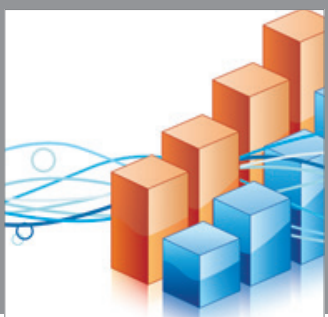

Advances in

Operations Research

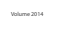

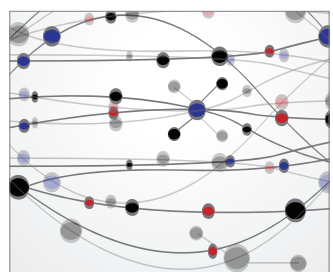

\section{The Scientific} World Journal
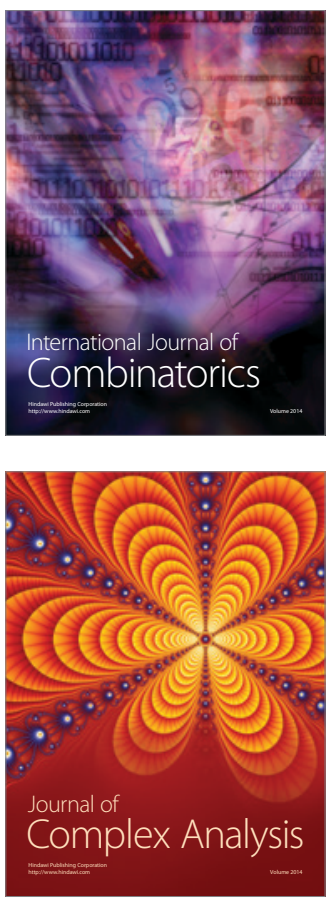

International Journal of

Mathematics and

Mathematical

Sciences
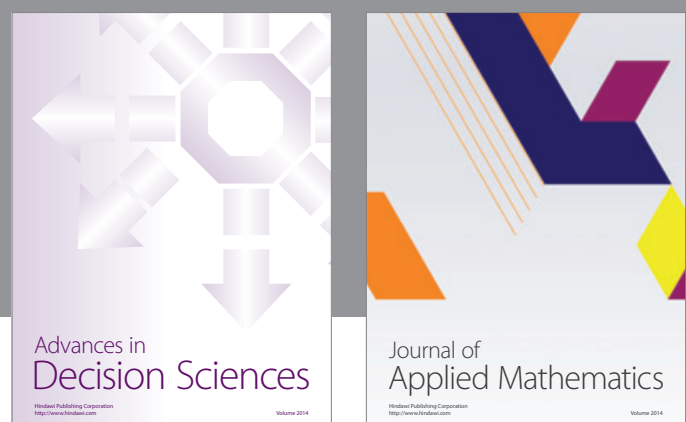

Journal of

Applied Mathematics
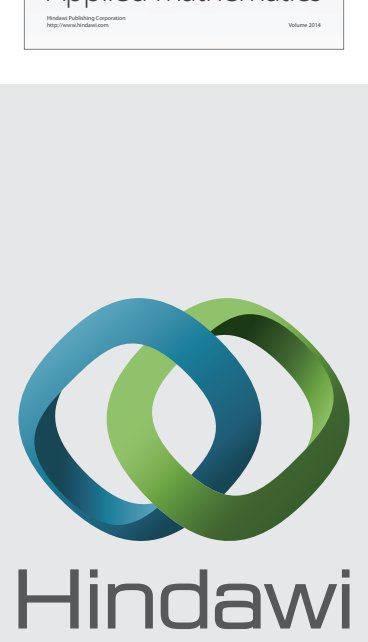

Submit your manuscripts at http://www.hindawi.com
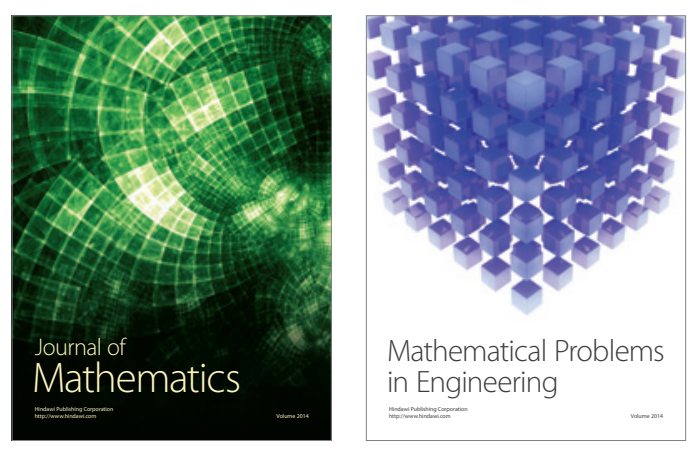

Mathematical Problems in Engineering
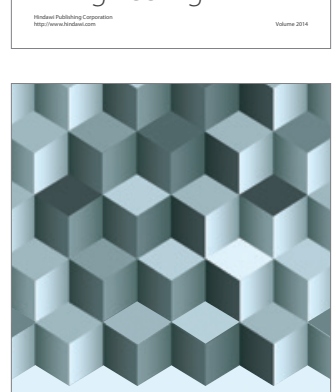

Journal of

Function Spaces
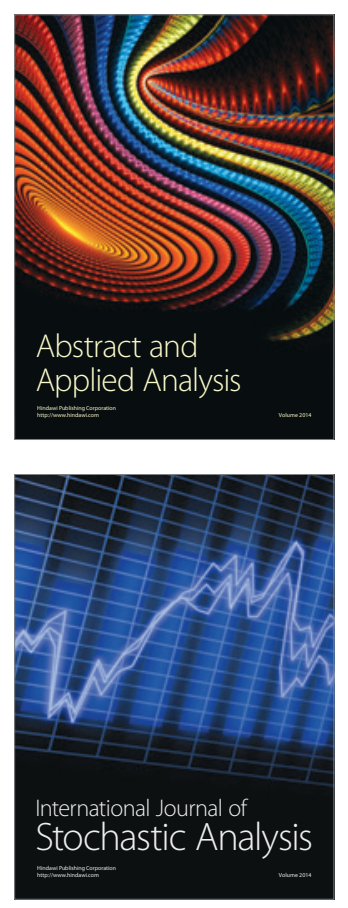

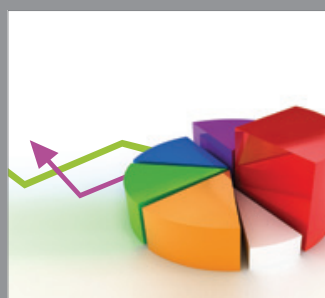

ournal of

Probability and Statistics

Promensencen
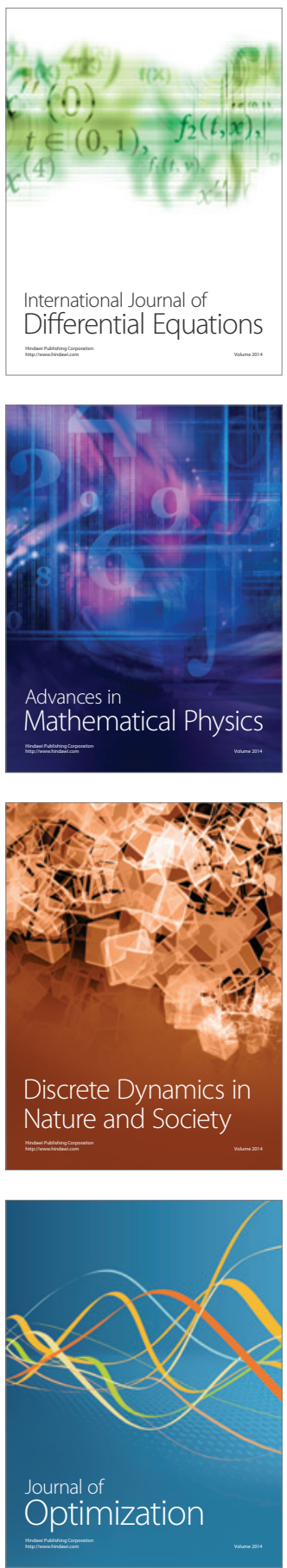